\title{
A Solution of the Dichromatic Model for Multispectral Photometric Invariance
}

\author{
Cong Phuoc Huynh ${ }^{1 *}$ and Antonio Robles-Kelly ${ }^{1,2}$ \\ ${ }^{1}$ School of Engineering, Australian National University, Canberra ACT 0200, Australia \\ ${ }^{2}$ National ICT Australia (NICTA) $\lesseqgtr$ Locked Bag 8001, Canberra ACT 2601, Australia
}

\begin{abstract}
In this paper, we address the problem of photometric invariance in multispectral imaging making use of an optimisation approach based upon the dichromatic model. In this manner, we cast the problem of recovering the spectra of the illuminant, the surface reflectance and the shading and specular factors in a structural optimisation setting. Making use of the additional information provided by multispectral imaging and the structure of image patches, we recover the dichromatic parameters of the scene. To do this, we formulate a target cost function combining the dichromatic error and the smoothness priors for the surfaces under study. The dichromatic parameters are recovered through minimising this cost function in a coordinate descent manner. The algorithm is quite general in nature, admitting the enforcement of smoothness constraints and extending in a straightforward manner to trichromatic settings. Moreover, the objective function is convex with respect to the subset of variables to be optimised in each alternating step of the minimisation strategy. This gives rise to an optimal closed-form solution for each of the iterations in our algorithm. We illustrate the effectiveness of our method for purposes of illuminant spectrum recovery, skin recognition, material clustering and specularity removal. We also compare our results to a number of alternatives.
\end{abstract}

Keywords: photometric invariance, multispectral imaging, dichromatic reflection model, reflectance.

\footnotetext{
${ }^{*}$ Corresponding author. E-mail: huynh@rsise.anu.edu.au. Tel: +61(2) 62676288

${ }^{\dagger}$ NICTA is funded by the Australian Government as represented by the Department of Broadband, Communications and the Digital Economy and the Australian Research Council through the ICT Centre of Excellence program.
} 


\section{Introduction}

In multispectral imaging, photometric invariants pose great opportunities and challenges in the areas of shape analysis and material identification [14]. This is due to the information-rich representation of the surface radiance acquired by multispectral and hyperspectral sensing devices, which deliver wavelength-indexed data in thousands of bands across a broad spectrum. Groundbased hyperspectral and multispectral imaging platforms, such as the Hyperspectral Image Intensified Camera System ${ }^{1}$ of OKSI, have recently become more commercially available. The advent of these commercial systems opens up opportunities for applications in areas such as material and object recognition and detection, biosecurity and surveillance. The ability to represent illumination and surface reflectance as a spectral signature allows greater accuracy and flexibility to interpret and distinguish colours than traditional trichromatic imagery. This is due to the robustness of spectral signatures to metamerism, i.e. trichromatic matches between materials which may be very different. In addition, hyperspectral imaging has been identified as a future direction in Computational Photography to reveal chemical or biological features for rendering and to provide high quality archival imaging [47].

Moreover, in computer vision, the modelling of surface reflectance is a topic of pivotal importance for purposes of surface analysis and image understanding. For instance, Nayar and Bolle [42] have used photometric invariants to recognise objects with different reflectance properties. This work builds on the one reported in [43], where a background to foreground reflectance ratio is introduced. In a related development, Dror et al. [16] have shown how surfaces may be classified from single images through the use of reflectance properties. Moreover, although shape-fromshading usually relies on the assumption of Lambertian reflectance [29], photometric correction or specularity subtraction may be applied as a preprocessing step to improve the results obtained.

The main bulk of work concentrates on the effects encountered on shiny or rough surfaces. For shiny surfaces, there are specular spikes and lobes which must be modelled. There have been several attempts to remove specularities from images of non-Lambertian objects. For instance Brelstaff and Blake [10] used a thresholding strategy to identify specularities on moving curved objects. Narasimhan et al. [41] have formulated a scene radiance model for the class of "separable" Bidirectional Reflectance Distribution Functions (BRDFs), which can be used to separate the model into material, object shape and lighting terms. More recently, Zickler et al. [61] introduced a

\footnotetext{
${ }^{1}$ For more information, see http://www.techexpo.com/WWW/opto-knowledge/prodhiicsi.html
} 
method for transforming the original RGB colour space into an illuminant-dependent colour space to obtain photometric invariants. Despite being effective, the application of these methods to multispectral imagery is somewhat limited since they are either constrained to trichromatic imagery or rely on the closed form of the Bidirectional Reflectance Distribution Function (BRDF).

Moreover, other alternatives elsewhere in the literature aiming at detecting and removing specularities either make use of additional hardware [42], impose constraints on the input images [39] or require colour segmentation [32]. Hence, they are not readily applicable to multispectral images, as there can be tens or even hundreds of bands for each pixel. Thus, any local operations, pre or postprocessing must be exercised with caution and in relation to neighbouring spectral bands so as to prevent spectral signature variation.

Specific to multispectral imagery, Healey and co-workers $[25,50,52]$ have addressed the problem of photometric invariance for material classification and mapping in aerial imaging as related to photometric artifacts induced by atmospheric effects and changing solar illumination. In [51], a method is presented for hyperspectral edge detection. The method is robust to photometric effects, such as shadows and specularities. In [1], a photometrically invariant approach was proposed based on the derivative analysis of the spectra. This local analysis of the spectra is intrinsic to the surface albedo. Nonetheless, the analysis in [1] was derived from the Lambertian reflection model and, hence, its not applicable to specular reflections. In [2], the same author derived a method to detect specular highlights in multispectral images by making use of the spectral derivative of the Fresnel reflection coefficient.

Since the recovery of illuminant and material reflectance are mutually interdependent, the problem here is closely related to colour constancy. Colour constancy is the ability to resolve the intrinsic material reflectance from their trichromatic colour images captured under varying illumination conditions. The research on colour constancy branches in two main trends, one of them relies on the statistics of illuminant and material reflectance, the other is drawn upon the physics-based analysis of local shading and specularity of the surface material.

In the statistics-based approaches, the colour of input images is often correlated against a collection of known illuminant chromaticities, such as those of Planckian light sources or black-body radiators. A few of these employ Bayes's rule $[8,9]$ to compute the best estimate from a posterior distribution by standard methods such as maximum a posteriori (MAP), minimum-mean-squared error (MMSE) or maximum local mass (MLM) estimation. The illuminant and surface reflectance spectra typically take the form of a finite linear model with a Gaussian basis. A well-known in- 
stance of this category is the Colour by Correlation method [5, 19], where a correlation matrix is built for a set of known plausible illuminants to characterise all the possible image colours (chromaticities) that can be observed. Gamut mapping methods [18, 20, 23], instead, gather the statistics of surface colours illuminated by a reference light source by taking the convex hull of the observed image colours. The rationale behind gamut mapping is to establish a linear map from the colour gamut of a given image to the canonical one, therefore recovering the illuminant colour of the given image by the inverse mapping. Simpler approaches assume some spatial statistics of image colours. For example, the Grey-World hypothesis [12] assumes that the spatial average of surface reflectances in a scene is achromatic, i.e. illuminant spectra can be estimated by taking the average of the sensor responses in the image. Similarly, the Grey-Edge hypothesis [57] states that the average edge difference in an image is achromatic.

Contrary to the statistics-based approaches, physics-based colour constancy analyses the physical processes by which light interacts with matter for the purpose of illuminant and surface reflectance estimation. The two famous corner stones of physics-based colour constancy are Land's retinex theory $[35,36]$ and the dichromatic reflection model [49]. Land's retinex theory has inspired several computational models of human colour constancy [7]. On the other hand, the dichromatic model describes reflected light as a combination of the body reflection and surface reflection (highlight), and therefore treating the illumination estimation problem as an analysis of highlights from shiny surfaces $[32,33,39,54]$. Based on this theory, the colours of all pixels of a uniform reflectance patch span a two-dimensional subspace of the colour space. Making use of this property, several authors have proposed illumination estimation techniques by computing the intersection of dichromatic planes [21, 55], or by introducing additional constraints such as assumed chromaticities of common light sources [22].

In contrast to the prior literature on colour constancy, the work presented here integrates the recovery of the illuminant, photometric invariants, i.e. the material reflectance, the shading and specularity factors from a single multispectral image in a unified optimisation framework. Not only the work extends the colour constancy problem from trichromatic to multispectral and hyperspectral imagery, but it also confers several advantages. By optimising the data closeness to the dichromatic model, the method is generally applicable to surfaces exhibiting both diffuse and specular reflection. In addition, our method makes no assumption on the parametric form or prior knowledge of the illuminant and surface reflectance spectra. This is in constrast to other approaches where assumptions are made on the chromaticities of common light sources or the finite linear 
model of illuminants and surface reflectance. Compared to other methods which make use of the dichromatic model $[21,22,55]$, our approach is able to perform well even on a small number of different material reflectance spectra. Furthermore, unlike the dichromatic plane-based methods for trichromatic imagery, our method does not require pre-segmented images as input. Instead, an automatic dichromatic patch selection process determines the uniform-albedo patches to be used for illuminant estimation. The noise perturbation analysis described in Section 5 shows that our illumination estimation method is more accurate than the alternatives and stable with respect to the number of surface patches used.

Moreover, the optimisation framework presented here is flexible and general in the sense that any regulariser on the image shading field can be incorporated into the method. In Section 3, we present two instances of robust regularisers for the smoothness of the shading field. The utility of regularisers has been a common practice in early vision problems [45] and particularly in Shapefrom-Shading [29], where regularisation together with occluding boundaries add supplementary constraints to make the underconstrained problem of inferring shape from shading well-posed [31]. Further, our objective function generalises prior colour constancy work $[21,48,55]$ based on leastsquares optimisation of the dichromatic model by controlling the surface smoothness through the use of regularisers. It is worth noting in passing that the shading factor in the dichromatic model reflects the angle between the incoming light direction and surface normals. Thus, the recovery of the shading factor by our optimisation method can be regarded as a pre-processing step for Lambertian Shape-from-Shading problems with spatially varying surface reflectance.

In this paper, we address the problem of recovering photometric invariants, namely material reflectance, through an estimation of the illumination power spectrum, the shading and specularity from a single multispectral image. Our proposed method assumes that the scene is uniformly illuminated. This assumption is common and valid for a wide range of situations, e.g. where the scene is illuminated by natural sunlight or a distant light source. Based upon the dichromatic reflection model [49], we cast the recovery problem as an optimisation one in a structural optimisation setting. Making use of the additional information provided by multispectral imaging and the structure of automatically selected image patches, we recover the dichromatic parameters of the scene. Since the objective function is convex with respect to each variable subset to be optimised upon, we can recover a closed-form solution which is iteration-wise optimal. We employ a quadratic surface smoothness error as a regulariser and show how a closed-form solution can be obtained when alternative regularisers are used. Later on, we show the successful application of 
our method to the tasks of illumination recovery and reflectance-based recognition. Although not originally designed for specularity removal, the method can also be applied to such an application with a milder level of success.

In Section 2, we present the target function employed in this paper. We elaborate further on the optimisation approach adopted here for the recovery of the parameters of the dichromatic reflection model. In Section 3 we show how smoothness constraints may be imposed upon the optimisation process. In Section 4, we provide a link between our method, which is hyperspectral in nature, and trichromatic imagery. In Section 5 we illustrate the utility of the method for the purposes of illuminant spectrum recovery, skin recognition, material clustering and specularity removal. This section mainly focuses on illumination recovery with supporting results from the skin recognition and material clustering experiments. In addition, it presents results for specularity removal purposes.

\section{Recovery of the Reflection Model Parameters}

Here, we present a structural approach based upon the processing of smooth surface-patches whose spectral reflectance is uniform over all those pixels they comprise. As mentioned earlier, the process of recovering the photometric parameters is based on an optimisation method which aims at reducing the difference between the estimate yielded by the dichromatic model and the input image. In this section, we commence by providing an overview of the dichromatic model as presented by Shafer [49]. Subsequently, we formulate a target minimisation function with respect to the model in [49] and derive an optimisation strategy based upon the radiance structure drawn from smooth image patches with uniform reflectance. Throughout the section, we also present our strategy for selecting patches used by the algorithm and describe in detail the coordinate descent optimisation procedure. This optimisation strategy is based upon interleaved steps aimed at recovering the light spectrum, the surface shading and surface reflectance properties so as to recover the optima of the dichromatic reflection parameters.

\subsection{The Dichromatic Reflection Model}

Throughout the paper, we employ the dichromatic model introduced by Shafer [49] so as to relate light spectral power, surface reflectance and surface radiance. This model assumes uniform illumi- 
nation across the spatial domain of the observed scene. Following this model, surface radiance is decomposed into a diffuse and a specular component. Let an object with surface radiance $I(\lambda, u)$ at pixel-location $u$ and wavelength $\lambda$ be illuminated by an illuminant whose spectrum is $L(\lambda)$. With these ingredients, the dichromatic model then becomes

$$
I(\lambda, u)=g(u) L(\lambda) S(\lambda, u)+k(u) L(\lambda)
$$

In Equation 1, the shading factor $g(u)$ governs the proportion of diffuse light reflected from the object and depends solely on the surface geometry. Note that, for a purely Lambertian surface, we have $g(u)=\cos (\overrightarrow{n(u)}, \overrightarrow{\mathcal{L}})$, i.e. the cosine of the angle between the surface normal $\overrightarrow{n(u)}$ and the light direction $\overrightarrow{\mathcal{L}}$. On the other hand, the factor $k(u)$ models surface irregularities that cause specularities in the scene. Using this model, we aim to recover the shading factor $g(u)$, the specular coefficient $k(u)$, the light spectrum $L(\lambda)$ and the spectral reflectance $S(\lambda, u)$ at location $u$ and wavelength $\lambda$ from the spectral radiance $I(\lambda, u)$ of the image.

\subsection{Target Function}

With the dichromatic model above, we proceed to define our target function for purposes of optimisation. Our algorithm takes as input a multispectral image whose pixel values correspond to the measurements of the spectral radiance $I(\lambda, u)$ indexed to the wavelengths $\lambda \in\left\{\lambda_{1}, \ldots \lambda_{n}\right\}$. As mentioned previously, our goal is fitting the observed data to the dichromatic model to recover the parameters $g(u), k(u)$ and $S(\lambda, u)$. In general, here we view the dichromatic cost function of a multispectral image $\mathcal{I}$ as the weighted sum of its dichromatic error and a regularisation term $R(u)$ for each image location. This is

$$
F(\mathcal{I}) \triangleq \sum_{u \in \mathcal{I}}\left[\sum_{i=1}^{n}\left[I\left(\lambda_{i}, u\right)-L\left(\lambda_{i}\right)\left(g(u) S\left(\lambda_{i}, u\right)+k(u)\right)\right]^{2}+\alpha R(u)\right]
$$

In equation 2, $\alpha$ is a constant that acts as a balancing factor between the dichromatic error and the regularisation term $R(u)$ on the right-hand side. The wavelength-independent regularisation term $R(u)$ is related to the surface shading and will be elaborated upon later.

For now, we focus our attention on the solution space of Equation 2. Note that minimising the cost $F(\mathcal{I})$ without further constraints is an underdetermined problem. This is due to the fact that, for an image with $n$ spectral bands containing $m$ pixels, we would have to minimise over $2 m+n+m \times n$ variables while having only $m \times n$ terms in the summation of Equation 2. However, 
we notice that this problem can be further constrained if the model is applied to smooth surfaces made of the same material, i.e. the albedo is uniform across the patch or image region under consideration. This imposes two constraints. Firstly, all locations on the surface share a common diffuse reflectance. Therefore, a uniform albedo surface $P$ is assumed to have the same reflectance for each pixel $u \in P, S\left(\lambda_{i}, u\right)=S_{P}\left(\lambda_{i}\right)$. Note that this constraint significantly reduces the number of unknowns $S\left(\lambda_{i}, u\right)$ from $m \times n$ to $N \times n$, where $N$ is the number of surface albedos in the scene. In addition, the smooth variation of the patch geometry allows us to formulate the regularisation term $R(u)$ in equation 2 as a function of the shading factor $g(u)$. In brief, smooth, uniform albedo surface patches naturally provide constraints so as to reduce the number of unknowns significantly while providing a plausible formulation of the regularisation term $R(u)$.

Following the rationale above, we proceed to impose constraints on the minimisation problem at hand. For a smooth, uniform-albedo surface patch $P \in \mathcal{I}$, we consider the following cost function

$$
F(P) \triangleq \sum_{u \in P}\left[\sum_{i=1}^{n}\left[I\left(\lambda_{i}, u\right)-L\left(\lambda_{i}\right)\left(g(u) S_{P}\left(\lambda_{i}\right)+k(u)\right)\right]^{2}+\alpha R(u)\right]
$$

As before, we have $S\left(\lambda_{i}, u\right)=S_{P}\left(\lambda_{i}\right)$, for all $u \in P$. Furthermore, the smoothness constraint on the patch implies that the shading factor $g(u)$ should vary smoothly across the pixels in $P$. This constraint can be effectively formulated by minimising the variation of gradient magnitude of the shading map. This, effectively, precludes discontinuities in the shading map of $P$ via the regularisation term

$$
R(u) \triangleq\left[\frac{\partial g(u)}{\partial x(u)}\right]^{2}+\left[\frac{\partial g(u)}{\partial y(u)}\right]^{2}
$$

where the variables $x(u)$ and $y(u)$ are the column and row coordinates, respectively, for pixel location $u$.

Thus, by making use of the set $\mathcal{P}$ of uniform-albedo patches in the image $\mathcal{I}$, we can recover the dichromatic model parameters by minimising the target function

$$
\begin{aligned}
F^{*}(\mathcal{I}) & \triangleq \sum_{P \in \mathcal{P}} F(P) \\
& =\sum_{P \in \mathcal{P}} \sum_{u \in P}\left[\sum_{i=1}^{n}\left[I\left(\lambda_{i}, u\right)-L\left(\lambda_{i}\right)\left(g(u) S_{P}\left(\lambda_{i}\right)+k(u)\right)\right]^{2}+\alpha R(u)\right]
\end{aligned}
$$

as an alternative to $F(\mathcal{I})$. 


\subsection{Light Spectrum Recovery}

\subsubsection{Homogeneous Patch Selection}

In the previous section, we formulated the recovery of the dichromatic model parameters as an optimisation procedure over the surface patch-set $\mathcal{P}$. In this section, we describe our method for automatically selecting uniform-albedo surface patches for the minimisation of the cost function in Equation 4. The automatic patch selection method presented here allows the application of our method to arbitrary images. It is worth noting that this contrasts with other methods elsewhere in the literature $[21,22,55,56]$, which are only applicable to pre-segmented images.

Our patch selection strategy is performed as follows. We first subdivide the image into patches of equal size in a lattice-like fashion. For each patch, we fit a two-dimensional hyperplane to the radiance vectors of the pixels in the patch. Next, we note that, in perfectly dichromatic patches, the wavelength-indexed radiance vector of each pixel lies perfectly in this hyperplane, i.e. the dichromatic plane. To allow for noise effect, we regard dichromatic patches as those containing a percentage of at most $t_{p}$ pixels whose radiance vectors deviate from their projection given by the Singular Value Decomposition (SVD) in [55]. We do this by setting a threshold $t_{a}$ on the angular deviation from the dichromatic plane, where $t_{p}$ and $t_{a}$ are global parameters.

However, not all these patches are useful for purposes of illumination spectrum recovery. This is due to the fact that perfectly diffuse surfaces do not provide any information regarding the illuminant spectrum. The reason being that, a spectral radiance vector space for this kind of surfaces is one-dimensional, spanned only by the wavelength-indexed diffuse radiance vector. On the other hand, the dichromatic model implies that the specularities have the same spectrum as the illuminant, where the specular coefficient can be viewed as a scaling factor solely dependent on the surface shading.

Thus, for the recovery of the dichromatic model parameters, we only use highly specular patches by selecting regions with the highest contrast amongst those deemed to have a uniform albedo. We recover the contrast of each patch by computing the variance of the mean radiance over the spectral domain. These highly specular patches provide a means to the recovery of the light spectrum. This is due to the fact that, for highly specular surface patches with uniform albedo, the surface diffuse radiance vector and the illuminant vector span a hyperplane in the radiance vector space. This is a well known property in colour constancy, where a number of approaches $[24,33,37]$ have employed subspace projection for purposes of light power spectrum recovery. 
$\mathbf{I}(u)$ : the spectral radiance vector at image pixel $u, \mathbf{I}(u)=\left[I\left(\lambda_{1}, u\right), \ldots I\left(\lambda_{n}, u\right)\right]^{T}$

$\mathbf{L}$ : the spectral power vector of the illuminant, $\mathbf{L}=\left[L\left(\lambda_{1}\right), \ldots L\left(\lambda_{n}\right)\right]^{T}$

$\mathbf{S}_{P}:$ the common spectral reflectance vector for each patch $P, \mathbf{S}_{P}=\left[S_{P}\left(\lambda_{1}\right), \ldots S_{P}\left(\lambda_{n}\right)\right]^{T}$

$\mathbf{g}_{P}:$ the shading map of all pixels in patch $P, \mathbf{g}_{P}=\left[g\left(u_{1}\right), \ldots g\left(u_{l}\right)\right]^{T}$ with $u_{1}, \ldots u_{l}$ being all the pixels in the patch $P$

$\mathbf{g} \quad$ : the shading map of all the patches, $\mathbf{g}=\left[\mathbf{g}_{P_{1}}^{T}, \ldots \mathbf{g}_{P_{r}}^{T}\right]^{T}$ where $P_{1}, \ldots P_{r}$ are all patches in $\mathcal{P}$

$\mathbf{k}_{P}:$ the specularity map of all pixels in patch $P, \mathbf{k}_{P}=\left[k\left(u_{1}\right), \ldots k\left(u_{l}\right)\right]^{T}$

$\mathbf{k} \quad:$ the specularity map of all the patches, $\mathbf{k}=\left[\mathbf{k}_{P_{1}}^{T}, \ldots \mathbf{k}_{P_{r}}^{T}\right]^{T}$

Figure 1: Notation for Section 2.3.2.

\subsubsection{Optimisation Procedure}

Making use of the notation in Figure 1, we now present the optimisation procedure employed in our method. Here, we adopt an iterative approach so as to find the variables $\mathbf{L}, \mathbf{S}_{P}, \mathbf{g}_{P}$ and $\mathbf{k}_{P}$ which yield the minimum of the cost function in Equation 4. At each iteration, we minimise the cost function with respect to $\mathbf{L}$ and the triplet $\left\{\mathbf{g}_{P}, \mathbf{k}_{P}, \mathbf{S}_{P}\right\}$ in separate steps.

The procedure presented here is, in fact, a coordinate descent approach [6] which aims at minimising the cost function. The step sequence of our minimisation strategy is summarised in the pseudocode of Algorithm 1. The coordinate descent approach comprises two interleaved minimisation steps. At each iteration, we index the dichromatic variables to iteration number $t$ and optimise the objective function, in interleaved steps, with respect to the two subsets of variables $\left\{\mathbf{g}_{P}, \mathbf{k}_{P}, \mathbf{S}_{P}\right\},\{\mathbf{L}\}$. Once the former variables are at hand, we can obtain optimal values for the latter ones. We iterate between these two steps until convergence is reached.

The algorithm commences by initialising the unknown light spectrum $L(\lambda)$ to an unbiased uniform illumination spectrum, as indicated in Line 1 of Algorithm 1. It terminates once the illuminant spectrum does not change, in terms of angle, by an amount beyond a preset global threshold $t_{\mathbf{L}}$ between two successive iterations. In the following two subsections we show that the two optimisation steps above can be employed to obtain the optimal values of the dichromatic parameters in closed form. 


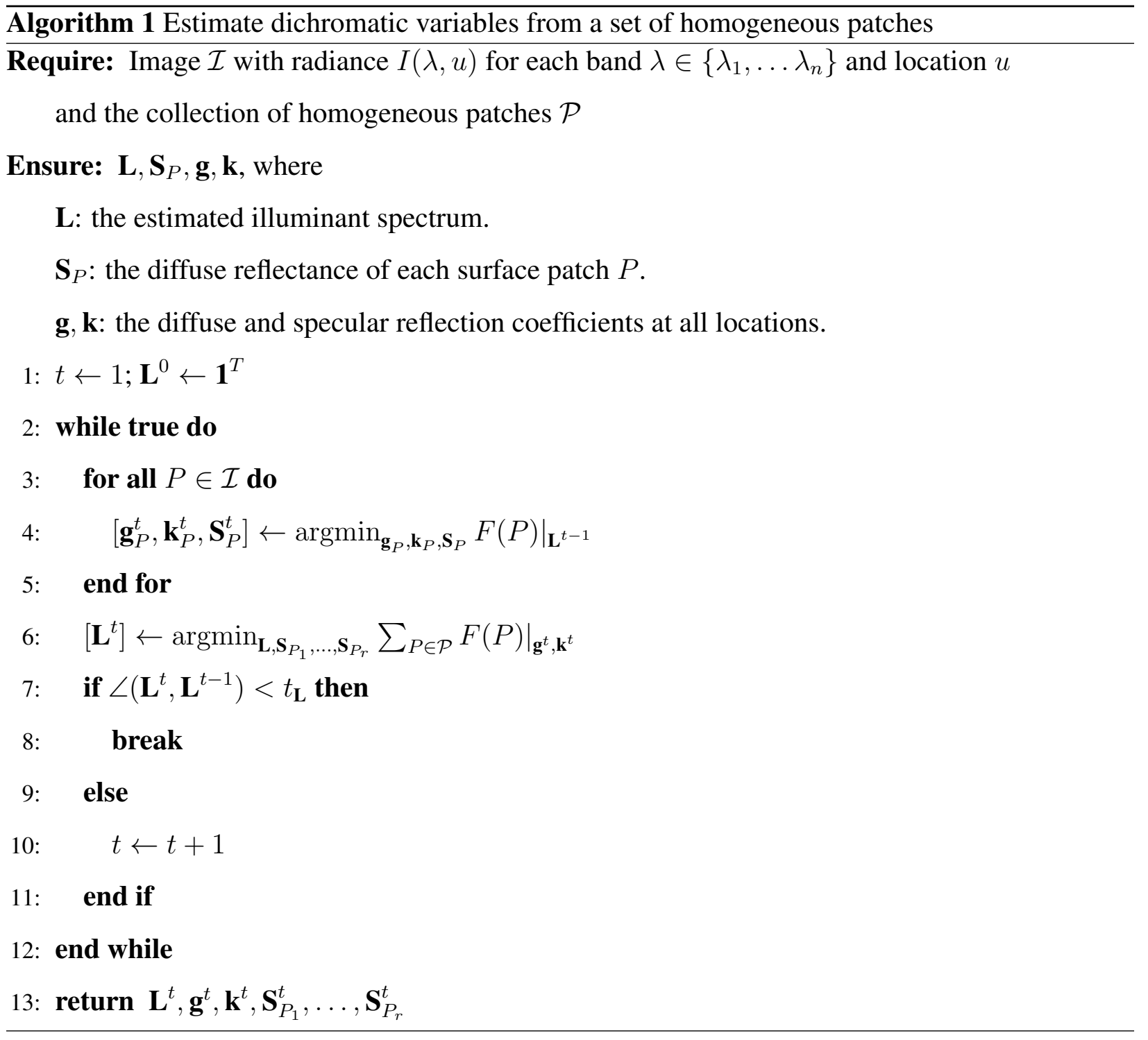

\section{Recovery of the Patch-set Surface Shading}

In the first step, we estimate the optimal surface reflectance and shading given the light spectrum $\mathbf{L}^{t-1}$ recovered at iteration $t-1$. This corresponds to Lines 3-5 in Algorithm 1. Note that, at iteration $t$, we can solve for the unknowns $\mathbf{g}_{P}^{t}, \mathbf{k}_{P}^{t}$ and $\mathbf{S}_{P}^{t}$ separately for each surface patch $P$. This is because, for each patch, these variables appear in a separate term in Equation 4. This step is, therefore, reduced to minimising

$$
\left.F(P)\right|_{\mathbf{L}^{t-1}}=\sum_{u \in P}\left[\left\|\mathbf{I}(u)-g(u) \mathbf{D}_{P}^{t-1}-k(u) \mathbf{L}^{t-1}\right\|^{2}+\alpha R(u)\right]
$$

where the diffuse radiance vector $\mathbf{D}_{P}^{t-1} \triangleq \mathbf{L}^{t-1} \bullet \mathbf{S}_{P}$ is the component-wise multiplication of the illuminant and surface reflectance spectra, and $\|$.$\| denotes the L_{2}$-norm of the argument vectors.

Note that the minimisation above involves $2|P|+n$ unknowns, where $|P|$ is the number of 
pixels in patch $P$. Hence, it becomes computationally intractable when the surface area is large. In practice, the selected patches need only be large enough so as to gather useful statistics from the radiance information. Moreover, as mentioned earlier, we can further reduce the degrees of freedom of the unknowns by noting that the spectral radiance vectors at all pixels in the same surface lie in a 2-dimensional subspace $Q \subset \mathbb{R}^{n}$, spanned by the diffuse radiance vector $\mathbf{D}_{P}^{t-1}$ and the light vector $\mathbf{L}^{t-1}$. This is a characteristic of the dichromatic model that has been widely utilised by prior work on colour constancy $[21,22,55,56]$.

Having all the pixel radiance vectors $\mathbf{I}(u)$ at hand, one can obtain the subspace $Q$ via Singular Value Decomposition (SVD). Denote the two basis vectors resulting from this SVD operation $\mathbf{z}_{1}$ and $\mathbf{z}_{2}$ and, accordingly, let the subspace be $Q=\operatorname{span}\left(\mathbf{z}_{1}, \mathbf{z}_{2}\right)$. Since $\mathbf{D}_{P}^{t-1} \in Q$, we can parameterise $\mathbf{D}_{P}^{t-1}$ up to scale as $\mathbf{D}_{P}^{t-1}=v \mathbf{z}_{1}+\mathbf{z}_{2}$.

Likewise, the light vector $\mathbf{L}^{t-1} \in Q$ can also be decomposed as $\mathbf{L}^{t-1}=w_{1} \mathbf{z}_{1}+w_{2} \mathbf{z}_{2}$, where the values of $w_{1}$ and $w_{2}$ are two known scalars. Furthermore, the dichromatic plane hypothesis also implies that, given the light vector $\mathbf{L}^{t-1}$ and the surface diffuse radiance vector $\mathbf{D}_{P}^{t-1}$, one can decompose any pixel radiance $\mathbf{I}(u)$ into a linear combination of the former two vectors. In other words,

$$
\begin{aligned}
\mathbf{I}(u) & =g(u) \mathbf{D}_{P}^{t-1}+k(u) \mathbf{L}^{t-1} \\
& =\left(g(u) v+k(u) w_{1}\right) \mathbf{z}_{1}+\left(g(u)+k(u) w_{2}\right) \mathbf{z}_{2}
\end{aligned}
$$

Having obtained the basis vectors $\mathbf{z}_{1}, \mathbf{z}_{2}$, we can compute the mapping of the pixel radiance $\mathbf{I}(u)$ onto the subspace $Q$. This is done with respect to this basis by means of projection so as to obtain the scalars $\tau_{1}(u), \tau_{2}(u)$ such that

$$
\mathbf{I}(u)=\tau_{1}(u) \mathbf{z}_{1}+\tau_{2}(u) \mathbf{z}_{2}
$$

Further, by equating the right hand sides of Equations 6 and 7, we obtain

$$
\begin{aligned}
g(u) & =\frac{w_{2} \tau_{1}(u)-w_{1} \tau_{2}(u)}{w_{2} v-w_{1}} \\
k(u) & =\frac{\tau_{2}(u) v-\tau_{1}(u)}{w_{2} v-w_{1}}
\end{aligned}
$$

From Equations 8 and 9, we note that $g(u)$ and $k(u)$ are univariate rational functions of $v$. Moreover, $\mathbf{D}_{P}^{t-1}$ is a linear function with respect to $v$. We also observe that the term $R(u)$ is only dependent on $g(u)$. Therefore, the objective function in Equation 5 can be reduced to a univariate 
rational function of $v$. Thus, substituting the Equations 8 and 9 into the first and second term on the right hand side of Equation 5, we have

$$
\begin{aligned}
\left.F(P)\right|_{\mathbf{L}^{t-1}}= & \sum_{u \in P}\left\|\mathbf{I}(u)-\frac{w_{2} \tau_{1}(u)-w_{1} \tau_{2}(u)}{w_{2} v-w_{1}}\left(v \mathbf{z}_{1}+\mathbf{z}_{2}\right)-\frac{\tau_{2}(u) v-\tau_{1}(u)}{w_{2} v-w_{1}} \mathbf{L}^{t-1}\right\|^{2} \\
& +\sum_{u \in P} \frac{\alpha}{\left(w_{2} v-w_{1}\right)^{2}}\left[\left(\frac{\partial m(u)}{\partial x(u)}\right)^{2}+\left(\frac{\partial m(u)}{\partial y(u)}\right)^{2}\right] \\
= & \sum_{u \in P} \frac{1}{\left(w_{2} v-w_{1}\right)^{2}} \|\left(\mathbf{I}(u) w_{2}-\left(w_{2} \tau_{1}(u)-w_{1} \tau_{2}(u)\right) \mathbf{z}_{1}-\tau_{2}(u) \mathbf{L}^{t-1}\right) v \\
& -\left(\mathbf{I}(u) w_{1}-\left(w_{2} \tau_{1}(u)-w_{1} \tau_{2}(u)\right) \mathbf{z}_{2}-\tau_{1}(u) \mathbf{L}^{t-1}\right) \|^{2} \\
& +\frac{\alpha}{\left(w_{2} v-w_{1}\right)^{2}} \sum_{u \in P}\left[\left(\frac{\partial m(u)}{\partial x(u)}\right)^{2}+\left(\frac{\partial m(u)}{\partial y(u)}\right)^{2}\right] \\
= & \sum_{u \in P}\left\|\frac{\mathbf{p}(u) v-\mathbf{q}(u)}{w_{2} v-w_{1}}\right\|^{2}+\frac{\alpha N}{\left(w_{2} v-w_{1}\right)^{2}} \\
= & \sum_{u \in P}\left\|\frac{\mathbf{p}(u)}{w_{2}}+\frac{\frac{w_{1}}{w_{2}} \mathbf{p}(u)-\mathbf{q}(u)}{w_{2} v-w_{1}}\right\|^{2}+\frac{\alpha N}{\left(w_{2} v-w_{1}\right)^{2}} \\
= & \sum_{u \in P} \frac{\|\mathbf{p}(u)\|^{2}}{w_{2}^{2}}+\frac{2}{w_{2} v-w_{1}} \sum_{u \in P}\left\langle\frac{\mathbf{p}(u)}{w_{2}}, \frac{w_{1}}{w_{2}} \mathbf{p}(u)-\mathbf{q}(u)\right\rangle \\
& +\frac{1}{\left(w_{2} v-w_{1}\right)^{2}}\left(\sum_{u \in P}\left\|\frac{w_{1}}{w_{2}} \mathbf{p}(u)-\mathbf{q}(u)\right\|^{2}+\alpha N\right)
\end{aligned}
$$

where $\langle.,$.$\rangle denotes the inner-product of two vectors, and$

$$
\begin{aligned}
m(u) & =w_{2} \tau_{1}(u)-w_{1} \tau_{2}(u) \\
\mathbf{p}(u) & =\mathbf{I}(u) w_{2}-\left(w_{2} \tau_{1}(u)-w_{1} \tau_{2}(u)\right) \mathbf{z}_{1}-\tau_{2}(u) \mathbf{L}^{t-1} \\
\mathbf{q}(u) & =\mathbf{I}(u) w_{1}-\left(w_{2} \tau_{1}(u)-w_{1} \tau_{2}(u)\right) \mathbf{z}_{2}-\tau_{1}(u) \mathbf{L}^{t-1} \\
N & =\sum_{u \in P}\left[\left(\frac{\partial m(u)}{\partial x(u)}\right)^{2}+\left(\frac{\partial m(u)}{\partial y(u)}\right)^{2}\right]
\end{aligned}
$$

Note that $\mathbf{p}(u), \mathbf{q}(u), w_{1}$ and $w_{2}$ are known given the vector $\mathbf{L}^{t-1}$. With the change of variable $r=\frac{1}{w_{2} v-w_{1}}$ we can write the right hand side of Equation 10 as a quadratic function of $r$ whose minimum is attained at

$$
r^{*}=-\frac{\sum_{u \in P}\left\langle\frac{\mathbf{p}(u)}{w_{2}}, \frac{w_{1}}{w_{2}} \mathbf{p}(u)-\mathbf{q}(u)\right\rangle}{\sum_{u \in P}\left\|\frac{w_{1}}{w_{2}} \mathbf{p}(u)-\mathbf{q}(u)\right\|^{2}+\alpha N}
$$

This gives the corresponding minimiser $v^{*}=\frac{1}{w_{2}}\left(\frac{1}{r^{*}}+w_{1}\right)$. Hence, given the illuminant spectrum $\mathbf{L}^{t-1}$, one can recover $\mathbf{g}_{P}, \mathbf{k}_{P}$ by substituting the optimal value of $v$ into Equations 8 and 9. The diffuse radiance component is computed as $\mathbf{D}_{P}^{t}=v^{*} \mathbf{z}_{1}+\mathbf{z}_{2}$, and the spectral reflectance at wavelength $\lambda$ is given by $\mathbf{S}_{P}^{t}(\lambda)=\frac{\mathbf{D}_{P}^{t}(\lambda)}{\mathbf{L}^{t-1}(\lambda)}$. 


\section{Recovery of the Illuminant Spectrum}

In the second step of each iteration $t$, we solve for $\mathbf{L}^{t}$ and $\mathbf{S}_{P_{1}}^{t}, \ldots, \mathbf{S}_{P_{r}}^{t}$ given $\mathbf{g}_{P}^{t}$ and $\mathbf{k}_{P}^{t}$. Since the second term $R(u)$ in Equation 4 is wavelength-independent, the optimisation problem in line 6 of Algorithm 1 can be reduced to minimising

$$
\begin{aligned}
\left.F^{*}(\mathcal{I})\right|_{\mathbf{g}^{t}, \mathbf{k}^{t}} & =\sum_{P \in \mathcal{P}} \sum_{u \in P}\left\|\mathbf{I}(u)-g^{t}(u) \mathbf{D}_{P}-k^{t}(u) \mathbf{L}\right\|^{2} \\
& =\sum_{P \in \mathcal{P}} \sum_{u \in P} \sum_{i=1}^{n}\left(I\left(\lambda_{i}, u\right)-g^{t}(u) D_{P}\left(\lambda_{i}\right)-k^{t}(u) L\left(\lambda_{i}\right)\right)^{2}
\end{aligned}
$$

where $\mathbf{D}_{P}=\mathbf{L} \bullet \mathbf{S}_{P}$

Since the objective function 12 is quadratic, and, therefore convex with respect to $\mathbf{L}$ and $\mathbf{D}_{P}$, the optimal values of these variables can be obtained by equating the respective partial derivatives of $\left.F^{*}(\mathcal{I})\right|_{\mathbf{g}^{t}, \mathbf{k}^{t}}$ to zero. These partial derivatives are given by

$$
\begin{aligned}
& \frac{\left.\partial F^{*}(\mathcal{I})\right|_{\mathbf{g}^{t}, \mathbf{k}^{t}}}{\partial L\left(\lambda_{i}\right)}=-2 \sum_{P \in \mathcal{P}} \sum_{u \in P}\left(I\left(\lambda_{i}, u\right)-g^{t}(u) D_{P}\left(\lambda_{i}\right)-k^{t}(u) L\left(\lambda_{i}\right)\right) k^{t}(u) \\
& \frac{\left.\partial F^{*}(\mathcal{I})\right|_{\mathbf{g}^{t}, \mathbf{k}^{t}}}{\partial D_{P}\left(\lambda_{i}\right)}=-2 \sum_{u \in P}\left(I\left(\lambda_{i}, u\right)-g^{t}(u) D_{P}\left(\lambda_{i}\right)-k^{t}(u) L\left(\lambda_{i}\right)\right) g^{t}(u)
\end{aligned}
$$

Equating the above equations to zero, we obtain

$$
\begin{aligned}
L\left(\lambda_{i}\right) & =\frac{\sum_{P \in \mathcal{P}} \sum_{u \in P}\left[k^{t}(u) I\left(\lambda_{i}, u\right)-g^{t}(u) k^{t}(u) D_{P}\left(\lambda_{i}\right)\right]}{\sum_{P \in \mathcal{P}} \sum_{u \in P}\left(k^{t}(u)\right)^{2}} \\
D_{P}\left(\lambda_{i}\right) & =\frac{\sum_{u \in P}\left[g^{t}(u) I\left(\lambda_{i}, u\right)-g^{t}(u) k^{t}(u) L\left(\lambda_{i}\right)\right]}{\sum_{u \in P}\left(g^{t}(u)\right)^{2}}
\end{aligned}
$$

From Equations 13 and 14, the illuminant spectrum can be solved in closed form as

$$
L^{*}\left(\lambda_{i}\right)=\frac{\sum_{P \in \mathcal{P}} \sum_{u \in P} k^{t}(u) I\left(\lambda_{i}, u\right)-\sum_{P \in \mathcal{P}}\left[\frac{\left(\sum_{u \in P} g^{t}(u) k^{t}(u)\right)\left(\sum_{u \in P} g^{t}(u) I\left(\lambda_{i}, u\right)\right)}{\sum_{u \in P}\left(g^{t}(u)\right)^{2}}\right]}{\sum_{P \in \mathcal{P}} \sum_{u \in P}\left(k^{t}(u)\right)^{2}-\sum_{P \in \mathcal{P}}\left[\frac{\left(\sum_{u \in P} g^{t}(u) k^{t}(u)\right)^{2}}{\sum_{u \in P}\left(g^{t}(u)\right)^{2}}\right]}
$$

\subsection{Shading, Reflectance and Specularity Recovery}

Note that, in the optimisation scheme above, we recover the reflectance, shading and specularity factors for pixels in each patch $P \in \mathcal{P}$ used for the recovery of the illuminant spectrum. This implies that, although we have only computed the variables $\mathbf{g}(u), \mathbf{k}(u)$ and $\mathbf{S}(., u)$ for pixel-sites $u \in \mathcal{P}$, we have been able to recover the illuminant spectrum $\mathbf{L}$. Since $\mathbf{L}$ is a global photometric variable in the scene, we can recover the remaining dichromatic variables making use of $\mathbf{L}$ in a 
straightforward manner. These include shading, reflectance and specularity factors for all image pixels.

For this purpose, we assume the input scene is composed of smooth surfaces with slowly varying reflectance. In other words, the neighbourhood of each pixel can be regarded as a locally smooth patch made of the same material, i.e. all the pixels in the neighbourhood share the same spectral reflectance. Given the illuminant spectrum, we can obtain the shading, specularity and reflectance of the neighbourhood at the pixel of interest by applying the procedure corresponding to line 4 in Algorithm 1. This corresponds to the application of the first of the two steps used in the optimisation method presented in the section above.

The pseudocode of this algorithm is summarised in Algorithm 2. Note that the assumption of smooth surfaces with slowly varying reflectance is applicable to a large category of scenes where surfaces have a low degree of texture, edges and occlusion. Following this assumption, the reflectance at each pixel is recovered as the shared reflectance of its surrounding patch. To estimate the shading and specularity, one can apply the closed-form formulae of these, as shown in Equations 8 and 9. These formulae yield exact solutions in the ideal condition, which requires that all the pixel radiance vectors lie in the same dichromatic hyperplane spanned by the illuminant spectrum and the diffuse radiance vector.

However, in practice, it is common for multi-spectral images to contain noise which breaks down this assumption and renders the above quotient expressions numerically unstable. Therefore, to enforce a smooth variation of the shading factor across pixels, we recompute the shading and specularity coefficients after obtaining the spectral reflectance. This is due to the observation that the reflectance spectrum is often more stable than the other two variables, i.e. shading and specularity factors. Specifically, one can compute the shading and specular coefficients as those resulting from the projection of pixel radiance onto the subspace spanned by the illuminant spectrum and the diffuse radiance spectrum vectors. 
$\overline{\text { Algorithm } 2 \text { Estimate the shading, specularity and reflectance of an image knowing the illuminant }}$ spectrum

Require: Image $\mathcal{I}$ with radiance $I(\lambda, u)$ for each band $\lambda \in\left\{\lambda_{1}, \ldots \lambda_{n}\right\}$

and the illuminant spectrum $\mathbf{L}$

Ensure: $\mathbf{g}(u), \mathbf{k}(u), \mathbf{S}(\lambda, u)$ where

$\mathbf{g}(u), \mathbf{k}(u)$ : the shading and specularity at pixel location $u$.

$\mathbf{S}(\lambda, u)$ : the diffuse reflectance of at pixel $u$ and wavelength $\lambda$.

1: for all $u \in \mathcal{I}$ do

2: $\quad \mathcal{N} \leftarrow$ Neighbourhood of $u$

3: $\left.\quad\left[\mathbf{g}_{\mathcal{N}}, \mathbf{k}_{\mathcal{N}}, \mathbf{S}_{\mathcal{N}}\right] \leftarrow \operatorname{argmin}_{\mathbf{g}_{\mathcal{N}}, \mathbf{k}_{\mathcal{N}}, \mathbf{S}_{\mathcal{N}}} F(P)\right|_{\mathbf{L}}$

4: $\quad \mathbf{S}(u) \leftarrow \mathbf{S}_{\mathcal{N}}$

5: end for

6: return $\mathbf{g}(u), \mathbf{k}(u), \mathbf{S}(., u)$

Similar to other photometric methods based on the dichromatic model, this framework breaks down when dichromatic hyper-plane assumption is violated, i.e. the illuminant spectrum is colinear to the diffuse radiance spectrum of the material. This renders the subspace spanned by the radiance spectra of the patch pixels to collapse to a 1-dimensional space. As a consequence, a Singular Value Decomposition of these radiance spectra does not succeed in finding two basis vectors of the subspace. Since the diffuse component is a product of the illuminant power spectrum and the material reflectance, this condition implies that the material has a uniform spectral reflectance. In other words, the failure case only happens when the input scene contains a single material with a uniform reflectance, i.e. one that resembles a shade of gray.

This failure case is very rare in practice. In fact, when the scene contains more than one material, as more uniform albedo patches are sampled from the scene, there are more opportunities to introduce the non-collinearity between the illuminant spectrum and surface diffuse radiance spectrum. In short, our method guarantees the recovery of dichromatic model parameters on scenes with more than one distinct albedo. 


\section{Imposing Smoothness Constraints}

In Section 2.2, we addressed the need of enforcing the smoothness constraint on the shading field $\mathbf{g}=\{\mathbf{g}(u)\}_{u \in \mathcal{I}}$ using the regularisation term $R(u)$ in Equation 2. In Equation 3, we present a regulariser that encourages the slow spatial variation of the shading field. There are two reasons for using this regulariser in the optimisation framework introduced in the previous sections. Firstly, it yields a closed-form solution for the surface shading and reflectance, given the illuminant spectrum. Secondly, it is reminiscent of smoothness constraints imposed upon shape from shading approaches and, hence, it provides a link between other methods in the literature, such as that in [59] and the optimisation method in the previous sections. However, we need to emphasise that the optimisation procedure above by no means implies that the framework is not applicable to alternative regularisers. In fact, our target function is flexible in the sense that other regularisation functions can be formulated dependent on the surface at hand.

In this section, we introduce a number of alternative regularisers on the shading field that are robust to noise and outliers and adaptive to the surface shading variation. To this end, we commence by introducing robust regularisers. We then present extensions based upon the surface curvature and the shape index.

To quantify the smoothness of shading, an option is to treat the gradient of the shading field as the smoothness error. In Equation 3, we have introduced a quadratic error function of the smoothness. However, in certain circumstances, enforcing the quadratic regulariser as introduced in Equation 2 causes the undesired effect of oversmoothing the surface. This well-known phenomenon has been experienced in a number of developments $[11,31]$ in the field of Shape from Shading. It is worth noting in passing that ample work exists in the literature addressing the over-smoothing tendency of quadratic regularisers used for enforcing smoothness constraints on gradients $[17,59,60]$.

As an alternative, we utilise kernel functions stemming from the field of robust statistics. Formally speaking, a robust kernel function $\rho_{\sigma}(\eta)$ quantifies an energy associated with both the residual $\eta$ and its influence function, i.e. measures sensitivity to changes in the shading field. Each residual is, in turn, assigned a weight as defined by an influence function $\Gamma_{\sigma}(\eta)$. Thus the energy is related to the first-moment of the influence function as $\frac{\partial \rho_{\sigma}(\eta)}{\partial \eta}=\eta \Gamma_{\sigma}(\eta)$. Table 1 shows the formulae for Tukey's bi-weight [26], Li's Adaptive Potential Functions [38] and Huber's Mestimators [30]. 


\begin{tabular}{|c|c|c|}
\hline Estimator & Robust kernel $\rho_{\sigma}(\eta)$ & Influence function $\Gamma_{\sigma}(\eta)$ \\
\hline Tukey & $\rho_{\sigma}(\eta)= \begin{cases}\sigma\left(1-\left(1-\left(\frac{\eta}{\sigma}\right)^{2}\right)^{3}\right) & \text { if }|\eta|<\sigma \\
\sigma & \text { otherwise }\end{cases}$ & $\Gamma_{\sigma}(\eta)= \begin{cases}\left(1-\left(\frac{\eta}{\sigma}\right)^{2}\right)^{2} & \text { if }|\eta|<\sigma \\
0 & \text { otherwise }\end{cases}$ \\
\hline $\mathrm{Li}$ & $\rho_{\sigma}(\eta)=\sigma\left(1-\exp \left(-\frac{\eta^{2}}{\sigma}\right)\right)$ & $\Gamma_{\sigma}(\eta)=\exp \left(-\frac{\eta^{2}}{\sigma}\right)$ \\
\hline Huber & $\rho_{\sigma}(\eta)= \begin{cases}\eta^{2} & \text { if }|\eta|<\sigma \\
2 \sigma|\eta|-\sigma^{2} & \text { otherwise }\end{cases}$ & $\Gamma_{\sigma}(x)=\left\{\begin{array}{cc}1 & \text { if }|\eta|<\sigma \\
\frac{\sigma}{|\eta|} & \text { otherwise }\end{array}\right.$ \\
\hline
\end{tabular}

Table 1: Robust kernels and influence functions.

\subsection{Robust Shading Smoothness Constraint}

Having introduced the above robust estimators, we proceed to employ them as regularisers for the target function. Here, several possibilities exist. One of them is to directly minimise the shading variation by defining robust regularisers with respect to the shading gradient. In this case, the regulariser $R(u)$ is given by the following formula

$$
R(u)=\rho_{\sigma}\left(\left|\frac{\partial g}{\partial x}\right|\right)+\rho_{\sigma}\left(\left|\frac{\partial g}{\partial y}\right|\right)
$$

Despite effective, the formula above still employs the gradient of the shading field as a measure of smoothness. In the next section, we explore the use of curvature as a measure of consistency.

\subsection{Curvature Consistency}

Alternatively, one can instead consider the intrinsic characteristics of the surface at hand given by its curvature. Specifically, Ferrie and Lagarde [17] have used the global consistency of principal curvatures to refine surface estimates in Shape from Shading. Moreover, ensuring the consistency of curvature directions does not necessarily imply a large penalty for discontinuities of orientation and depth. Therefore, this measure can avoid oversmoothing, which is a drawback of the quadratic smoothness error.

The curvature consistency can be defined on the shading field by treating it as a manifold. To commence, we define the structure of the shading field using its Hessian matrix

$$
H=\left(\begin{array}{cc}
\frac{\partial^{2} g}{\partial x^{2}} & \frac{\partial^{2} g}{\partial x \partial y} \\
\frac{\partial^{2} g}{\partial y \partial x} & \frac{\partial^{2} g}{\partial y^{2}}
\end{array}\right)
$$

The principal curvatures of the manifold are hence defined as the eigenvalues of the Hessian matrix. Let these eigenvalues be denoted by $\kappa_{1}$ and $\kappa_{2}$, where $\kappa_{1} \geq \kappa_{2}$. Moreover, we can use the 
principal curvatures to describe local topology using the Shape Index [34] defined as follows

$$
\phi=\frac{2}{\pi} \arctan \left(\frac{\kappa_{1}+\kappa_{2}}{\kappa_{1}-\kappa_{2}}\right)
$$

The observation above is important because it permits casting the smoothing process of the shading field as a weighted mean process, where the weight assigned to a pixel is determined by the similarity in local topology, i.e. the shape index, about a local neighbourhood. Effectively, the idea is to favour pixels in the neighbourhood that belong to the same or similar shape class as the pixel of interest. This is an improvement over the quadratic smoothness term defined in Equation 3 because it avoids the indiscriminate averaging of shading factors across discontinuities. That is, it is by definition edge preserving.

For each pixel $u$, we consider a local neighbourhood $\mathcal{N}$ around $u$ and assign a weight to each pixel $u^{*}$ in the neighbourhood as $w\left(u^{*}\right)=\exp \left(-\frac{\left(\phi\left(u^{*}\right)-\mu_{\phi}(\mathcal{N})\right)^{2}}{2 \sigma_{\phi}^{2}(\mathcal{N})}\right)$, where $\mu_{\phi}(\mathcal{N})$ and $\sigma_{\phi}(\mathcal{N})$ are the mean and standard deviation of shape index over the neighbourhood $\mathcal{N}$. Using this weighting process, we obtain an adaptive weighted mean regulariser as follows

$$
R(u)=\left(g(u)-\frac{\sum_{u^{*} \in \mathcal{N}} w\left(u^{*}\right) g\left(u^{*}\right)}{\sum_{u^{*} \in \mathcal{N}} w\left(u^{*}\right)}\right)^{2}
$$

This approach can be viewed as an extension of the robust regulariser function with a fixed kernel, presented in Equation 16. To regulate the level of smoothing applied to a neighbourhood, we consider the shape index statistics [34] so as to adaptively change the width of the robust kernel. The rationale behind adaptive kernel widths is that a neighbourhood with a great variation of shape index requires stronger smoothing than one with a smoother variation. The regulariser function is exactly the same as Equation 16, except for the kernel width which is defined pixel-wise as

$$
\sigma(u)=\exp \left(-\left(\frac{1}{K_{\phi}|\mathcal{N}|} \sum_{u^{*} \in \mathcal{N}}\left(\phi\left(u^{*}\right)-\phi(u)\right)^{2}\right)^{1 / 2}\right)
$$

where $\mathcal{N}$ is a neighbourhood around the pixel $u,|\mathcal{N}|$ is the cardinality of $\mathcal{N}$ and $K_{\phi}$ is a normalisation term.

With the above formulation of the kernel width, it can be observed that a significant variation of the shape index within the neighbourhood corresponds to a small kernel width, causing the robust regulariser to produce heavy smoothing. In contrast, when the shape index variation is small, a lower level of smoothing occurs due to a wider kernel width. 
Note that the use of the robust regularisers introduced earlier in this section as an alternative to the quadratic regulariser does not preclude the applicability of the optimisation framework described in Section 2.3.2. In fact, the change of regulariser only affects the formulation of the target function in Equation 10, in which the shading factor $g(u)$ can be expressed as a univariate function as given in Equation 8. Since all the above robust regularisers are only dependent on the shading factor, the resulting target function is still a function of the variable $r \triangleq \frac{1}{w_{2} v-w_{1}}$. Further, by linearisation of the robust regularisers, one can still numerically express the regulariser as a quadratic function of the variable $r$. Subsequently, the closed-form solution presented earlier stands as originally described.

\section{Adaptation to Trichromatic Imagery}

In this section, we show how to utilise the optimisation method above to recover the dichromatic parameters from trichromatic images. To this end, we transform the dichromatic model for multispectral images into one for trichromatic imagery. Let us denote the spectral sensitivity function of the trichromatic sensor $c$ (where $c \in\{R, G, B\}$ ) by $C_{c}(\lambda)$. The response of the sensor $c$ to the spectral irradiance arriving at the location $u$ is given by $I_{c}(u)=\int_{\Omega} E(\lambda, u) C_{c}(\lambda) d \lambda$, where $E(\lambda, u)$ is the image irradiance and $\Omega$ is the spectrum of the incoming light. Furthermore, it is well-known that the image irradiance is proportional to the scene radiance $I(\lambda, u)$, i.e. $E(\lambda, u)=K_{\text {opt }} \cos ^{4} \beta(u) I(\lambda, u)$, where $\beta(u)$ is the angle of incidence of the incoming light ray on the lens and $K_{\text {opt }}$ is a constant only dependent on the optics of the lens [28]. Hence, we have

$$
\begin{aligned}
I_{c}(u) & =K_{\text {opt }} \cos ^{4} \beta(u) \int_{\Omega} I(\lambda, u) C_{c}(\lambda) d \lambda \\
& =K_{\text {opt }} \cos ^{4} \beta(u) \int_{\Omega}(g(u) L(\lambda) S(\lambda, u)+k(u) L(\lambda)) C_{c}(\lambda) d \lambda \\
& =K_{\text {opt }} \cos ^{4} \beta(u) \int_{\Omega} L(\lambda) S(\lambda, u) C_{c}(\lambda) d \lambda+K_{\text {opt }} \cos ^{4} \beta(u) k(u) \int_{\Omega} L(\lambda) C_{c}(\lambda) d \lambda \\
& =g^{*}(u) D_{c}(u)+k^{*}(u) L_{c}
\end{aligned}
$$

where $g^{*}(u)=K_{\text {opt }} \cos ^{4} \beta(u) g(u)$ and $k^{*}(u)=K_{\text {opt }} \cos ^{4} \beta(u) k(u)$.

Here we notice that $D_{c}(u)=\int_{\Omega} L(\lambda) S(\lambda, u) C_{c}(\lambda) d \lambda$ and $L_{c}(u)=\int_{\Omega} L(\lambda) C_{i}(\lambda) d \lambda$ are the $c$ component of the surface diffuse colour corresponding to the location $u$ and of the illuminant colour, respectively. 
The dichromatic cost function for the trichromatic image $\mathcal{I}$ of a scene is formulated as

$$
F(\mathcal{I}) \triangleq \sum_{u \in \mathcal{I}}\left[\sum_{c \in\{R, G, B\}}\left[I_{c}(u)-\left(g^{*}(u) D_{c}(u)+k^{*}(u) L_{c}\right)\right]^{2}+\alpha R(u)\right]
$$

where $R(u)$ is a spatially varying regularisation term, as described in Equation 2.

It is worth noticing that the cost function in Equation 20 is a special case of Equation 2, where $n=3$. Hence, the method of recovering the dichromatic parameters, as elaborated upon in Sections 2.3.1 and 2.3.2 can be applied to this case in order to recover the trichromatic diffuse colour $\mathbf{D}(u)=\left[D_{R}(u), D_{G}(u), D_{B}(u)\right]^{T}$ and illuminant colour $\mathbf{L}=\left[L_{R}, L_{G}, L_{B}\right]^{T}$, as well as the shading and specular factors $g(u)$ and $k(u)$ up to a multiplier.

\section{Experiments}

In this section, we perform experiments on a number of image databases so as to verify the accuracy of the recovered dichromatic parameters. Our datasets include indoor and outdoor multispectral and RGB images with uniform and cluttered backgrounds, under natural and artificial lighting conditions. For this purpose, we have acquired in-house two multi-spectral image databases captured in the visible and near-infrared ranges. These consist of indoor images taken under artificial light sources and outdoor images under natural sunlight and skylight. From these two databases, two trichromatic image databases are synthesized for the spectral sensitivity functions of a Canon 10D and a Nikon D70 camera sensor and the CIE standard RGB colour matching functions [15]. Apart from these databases, we have also compared the performance of our algorithm with the alternatives on the benchmark dataset reported by Barnard et al. in [3].

The indoor database includes images of 51 human subjects, each captured under one of 10 directional light sources with varying directions and spectral power. The light sources are divided into two rows. The first of these is placed above the camera system and the second one at the same height as the cameras. The main direction of the lights is adjusted so as to point towards the centre of the scene. The imagery has been acquired using a pair of OKSI Turnkey Hyperspectral Cameras. These cameras are equipped with Liquid Crystal Tunable Filters which allow multispectral images to be resolved up to $10 \mathrm{~nm}$ in both the visible $(430-720 \mathrm{~nm})$ and the near infrared (650-990 $\mathrm{nm}$ ) wavelength ranges. To obtain the ground truth illuminant spectrum for each image, we have measured the average radiance reflected from a white calibration target, i.e. a LabSphere 
Spectralon, illuminated by the light sources under consideration. Using the same camera system and calibration target, we have captured the outdoor images of a paddock from four different viewpoints, each from seven different viewing angles at different times of the day.

In the following experiments, we explore the utility of the recovered parameters of the dichromatic model for multiple applications. Throughout these experiments, our method is shown to be most successful in delivering competitive performance for illumination spectrum recovery and material recognition purposes. Therefore, we present the main bulk of the experiments in Section 5.1, where we demonstrate the effectiveness of our method for illumination spectrum recovery. In Section 5.2, we present results for skin recognition and material clustering tasks. The purpose of the section is two-fold, one of which is to assess the robustness of the recovered reflectance for material recognition, the other is to reaffirm the accuracy of the illumination spectrum recovery results presented in Section 5.1. Lastly, we explore the use of the recovered shading and specularity coefficients for specularity removal in Section 5.3. Note that, although the method was not originally designed for specularity removal, it may also be applied for such a purpose with moderate success.

\subsection{Illumination Spectrum Recovery}

For our experiments on illumination spectra recovery, we compare the results yielded by our method to those delivered by the colour constancy method proposed by Finlayson and Schaefer [21]. In [21], illuminant colours are estimated based on the dichromatic model without prior assumptions on the illuminant statistics. Although their experiments were performed on trichromatic imagery, this method can be adapted to multispectral data in a straightforward manner. Their approach relies on the dichromatic plane hypothesis. This is, that the dichromatic model implies a two-dimensional colour space of pixels in patches with homogeneous reflectance. Utilising this idea, illumination estimation is cast as an optimisation problem so as to maximise the total projection length of the light colour vector on all the dichromatic planes. Geometrically, this approach predicts the illuminant colour as the intersection of dichromatic planes, which may lead to a numerically unstable solution when the angle between dichromatic planes are small.

Finlayson and Schaefer's method can be adapted to multispectral images as follows. First, we employ our automatic patch selection method to provide homogeneous patches as input for their colour constancy algorithm. Secondly, we solve the eigen-system of the sum of projection matrices on the dichromatic planes. The light colour vector is the eigenvector corresponding to the largest 
eigenvalue.

The other alternative used here is akin to the spectrum deconvolution approach proposed by Sunshine et al. [53] to recover the absorption bands characteristic of the surface material chemistry. This method makes use of the upperbound envelope of a reflectance spectrum, also known as its continuum, which can be regarded as a reflectance spectrum without any absorption feature. For illuminant recovery, we view the estimated illuminant spectrum as the continuum of the radiance spectra at all the pixels. The work in [53] assumes that the continuum is a linear function of the wave number, i.e. the reciprocal of wavelength, on the log reflectance scale. Making use of this assumption, it then fits this parametric form to the continuum of the radiance spectra to recover the illuminant. Note that the resulting illuminant does not rely on patch selection and is therefore independent of the number of patches.

The section is organised as follows. We commence by providing results on hyperspectral imagery. We then turn our attention to light colour recovery in trichromatic imagery. We conclude the section by providing a noise perturbation analysis.

\subsubsection{Multispectral Light Spectrum Recovery}

As mentioned above, we first focus our attention on the use of our dichromatic parameter recovery algorithm for illuminant spectrum estimation in hyperspectal imagery. To this end, we have performed experiments using 1, 5, 10,20,30, 40 and 50 automatically selected patches of uniform albedo. Each patch has a size of $20 \times 20$ pixels. The accuracy of light spectrum recovery is measured as the Euclidean deviation angle between the estimated and ground truth spectrum in $n$ dimensions, where $n$ is the number of sampled wavelengths. These results are then compared against those obtained by the method of Finlayson and Schaefer [21] and Sunshine et al.'s [53] on the same number of patches.

Table 2 shows the means and standard deviations of the angular errors, in degrees, over all images in the indoor face database versus of the number of selected patches in both, the visible and infrared spectral ranges. Similar statistics are plotted in Figure 2, with the means and standard deviations of the angular errors represented by the midpoint and the length of the error bars. Again, note that the method of Sunshine et al. [53] is independent of the number of selected patches.

The results are reported with a weight $\alpha=100000$ assigned to the regularisation term in Equation 2. In this experiment, the regularisation term is defined to be the smoothness of shading 


\begin{tabular}{|c|c|c|c|c|c|c|}
\hline \multirow{2}{*}{$\begin{array}{c}\text { No. } \\
\text { patches }\end{array}$} & \multicolumn{3}{|c|}{ Visible spectrum } & \multicolumn{3}{c|}{ Near-infrared spectrum } \\
\cline { 2 - 7 } & Our method & F \& S & Sunshine & Our method & F \& S & Sunshine \\
\hline 1 & $17.25 \pm 6.07$ & $20.55 \pm 10.53$ & $9.52 \pm 1.40$ & $7.41 \pm 5.37$ & $25.50 \pm 8.88$ & $19.9 \pm 2.24$ \\
\hline 5 & $5.62 \pm 5.54$ & $7.52 \pm 4.90$ & - & $6.44 \pm 5.09$ & $7.00 \pm 4.56$ & - \\
\hline 10 & $5.81 \pm 5.63$ & $7.42 \pm 4.19$ & - & $6.65 \pm 5.05$ & $6.98 \pm 4.04$ & - \\
\hline 20 & $6.21 \pm 5.22$ & $7.37 \pm 3.88$ & - & $6.87 \pm 4.95$ & $7.03 \pm 3.58$ & - \\
\hline 30 & $6.49 \pm 5.53$ & $7.32 \pm 3.65$ & - & $7.56 \pm 5.18$ & $7.06 \pm 3.46$ & - \\
\hline 40 & $6.66 \pm 5.86$ & $7.29 \pm 3.54$ & - & $7.85 \pm 5.07$ & $7.06 \pm 3.39$ & - \\
\hline 50 & $6.82 \pm 5.84$ & $7.26 \pm 3.50$ & - & $7.90 \pm 5.25$ & $7.09 \pm 3.33$ & - \\
\hline
\end{tabular}

Table 2: Accuracy versus the number of patches used for our illuminant estimation method on the multispectral facial image database captured under both the visible and near-infrared spectra, in degrees, compared to Finlayson and Schaefer's method (F \& S) and Sunshine et al.'s method.

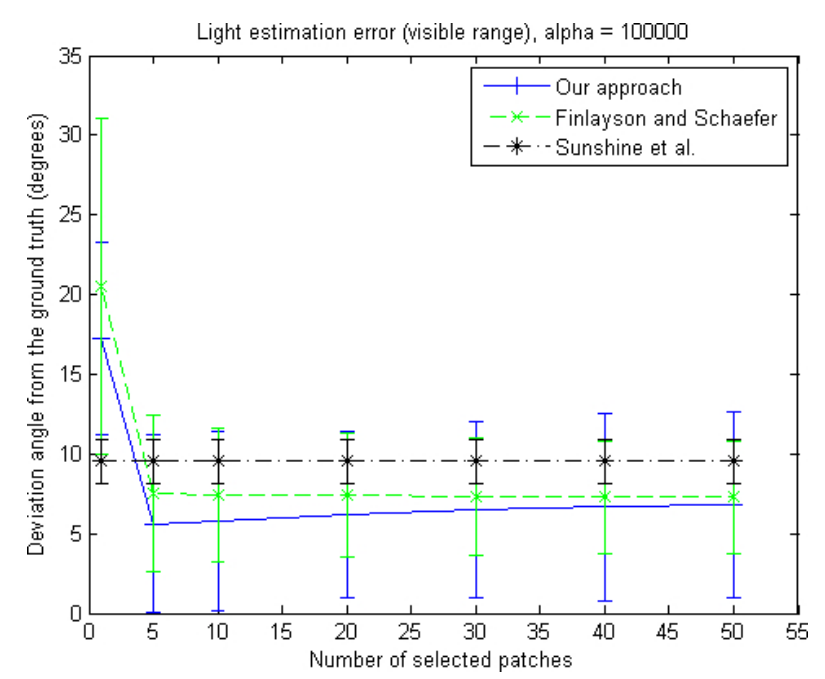

(a) Visible spectrum

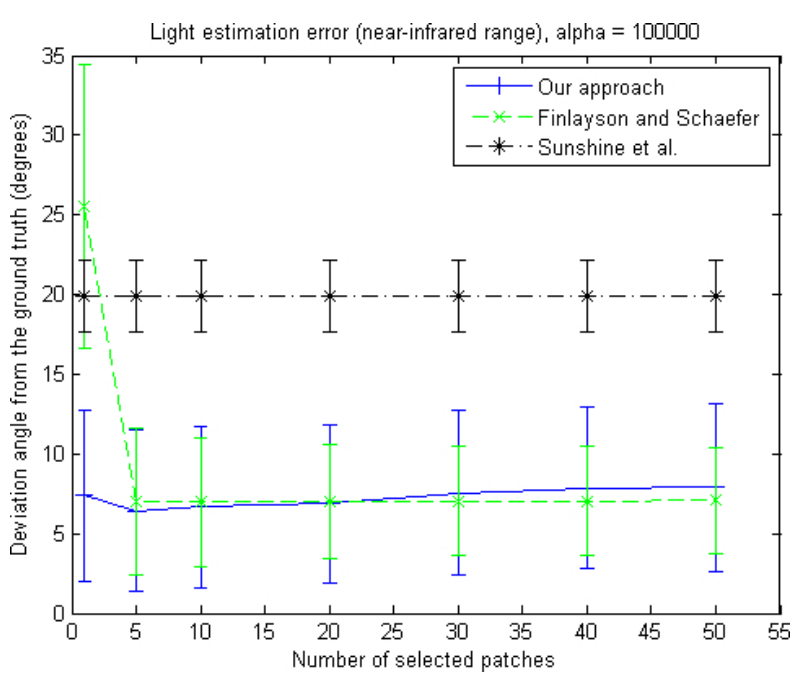

(b) Near-infrared spectrum

Figure 2: Accuracy versus number of patches used of our illuminant estimation method on the multispectral facial image database, in degrees, compared to Finlayson and Schaefer's method (F \& S) and Sunshine et al.'s method. The results for both the visible (left) and near-infrared ranges (right) are shown.

variation, as shown in Equation 3. To obtain an optimal value of $\alpha$, we perform a procedure similar to the grid search employed in cross validation. The procedure involves applying our algorithm on a randomly sampled portion of the database several times for different parameter values and then selecting the value that yields the highest overall performance.

As shown in Table 2, our algorithm achieves a higher accuracy than the alternative methods when using no more than 20 homogeneous patches for both spectral ranges. It is noticeable that even with 
a single patch, our method still significantly outperforms Finlayson and Schaefer's method [21]. This observation is consistent with the well-known fact that the dichromatic plane method and its variants require at least two homogeneous surfaces to compute the intersection between the dichromatic planes. In addition, the angular error of the estimated illuminant decreases as the number of patches increases from 1 to 5 . However, as the number of patches grows beyond 5 , the angular error of our method tends to increase. Although our methods remains more accurate than Finlayson and Schaefer's method in the visible range, its accuracy is slightly lower than the latter with more than 20 patches in the near-infrared range. Nonetheless, our method is able to achieve a reasonable estimate with a small number of homogeneous patches. Lastly, we can conclude that Sunshine et al.'s method [53] is, in general, inferior to the other two.

To illustrate the statistics in Table 2, we show, in Figure 3, the plots of the estimated spectra of a light source illuminating an indoor scene. The plots show spectra in both the visible (left column) and infra-red (right column) ranges. In each row, from top to bottom, we show the results yielded by our method and the alternatives using a different number of homogeneous patches for illuminant estimation. As before, we show the plots for 1, 5, 10, 40 and 50 patches. The ground truth spectra, the spectra estimated by our method, Finlayson and Schaefer's [21] and Sunshine et al.'s method [53] are drawn in red, blue, green and magenta, respectively. Note that the highest value of each spectra is normalised to unity.

This visual illustration is consistent with a common trend in Table 2, that increasing the number of patches from 1 to 5 yields a significant improvement of accuracy for illumination estimation. Noticeably, our method outperforms the alternatives in recovering illumination spectra in the visible range. Meanwhile, its performance for the near-infrared range is comparable to Finlayson and Schaefer's [21] and is better than Sunshine et al.'s method [53]. Moreover, our method is more robust than the others even when it uses a single homogeneous patch for light estimation.

In Table 3 and Figure 4, we show the accuracy of the recovered spectrum of natural sunlight illuminating the outdoor scene in our dataset. Here our algorithm is applied with a regularisation weight $\alpha=10000000$. With this setting, our method always outperforms the alternatives in the visible range. Using 20 or more randomly selected patches is sufficient for our method to improve performance over Finlayson and Schaefer's [21] on the near-infrared images. As before, our algorithm significantly outperforms the alternative methods in the case of a single uniform albedo patch. Figure 4 also illustrates the stability of our method with respect to the increase in the number of selected patches. It is also noticeable that the accuracy of all the algorithms for the outdoor 

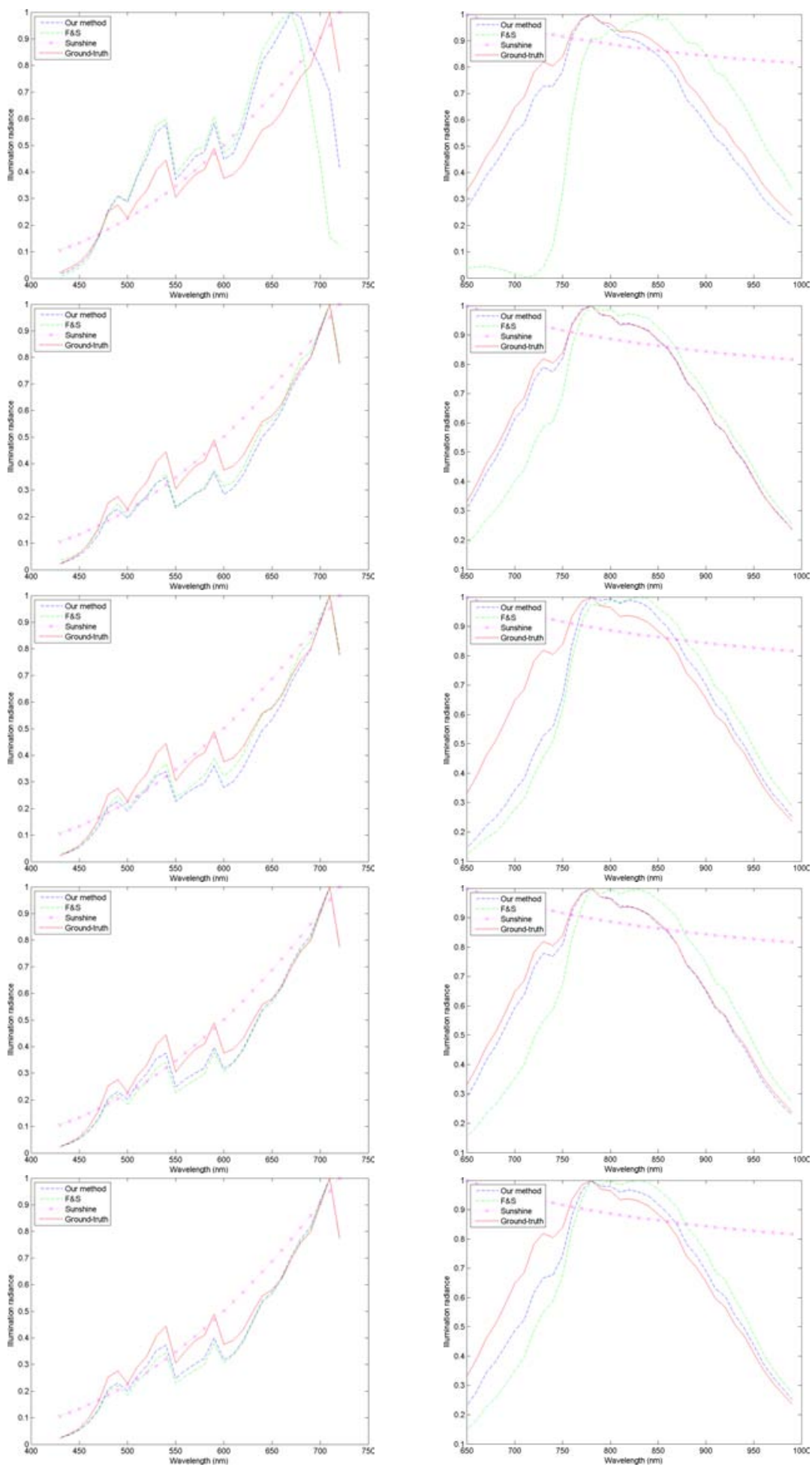

Figure 3: Ground truth illuminant spectra and those estimated by our method and the alternatives from the image of a human subject in the dataset illuminated by an high-oblique light direction (from above and to the left of the camera center). Here we show the estimated illuminant spectra in both the visible (left column) and near-infrared (right column). From top to bottom: the spectra estimated using 1, 5, 10, 40 and 50 patches from the image. 


\begin{tabular}{|c|c|c|c|c|c|c|}
\hline \multirow{2}{*}{$\begin{array}{c}\text { No. } \\
\text { patches }\end{array}$} & \multicolumn{3}{|c|}{ Visible spectrum } & \multicolumn{3}{c|}{ Near-infrared spectrum } \\
\cline { 2 - 7 } & Our method & F \& S & Sunshine & Our method & F \& S & Sunshine \\
\hline 1 & $13.93 \pm 2.83$ & $22.17 \pm 5.84$ & $17.91 \pm 5.43$ & $9.35 \pm 4.63$ & $29.46 \pm 8.18$ & $17.13 \pm 2.18$ \\
\hline 5 & $13.89 \pm 1.25$ & $14.06 \pm 1.47$ & - & $8.08 \pm 3.38$ & $6.97 \pm 2.59$ & - \\
\hline 10 & $13.80 \pm 1.17$ & $14.09 \pm 1.35$ & - & $8.47 \pm 2.58$ & $7.31 \pm 1.80$ & - \\
\hline 20 & $14.00 \pm 1.43$ & $14.03 \pm 1.33$ & - & $7.07 \pm 2.51$ & $7.20 \pm 1.38$ & - \\
\hline 30 & $13.69 \pm 1.53$ & $14.00 \pm 1.23$ & - & $6.98 \pm 2.41$ & $7.48 \pm 1.50$ & - \\
\hline 40 & $13.44 \pm 2.09$ & $13.99 \pm 1.22$ & - & $7.22 \pm 2.48$ & $7.69 \pm 1.59$ & - \\
\hline 50 & $13.85 \pm 1.48$ & $13.97 \pm 1.24$ & - & $7.33 \pm 2.48$ & $7.74 \pm 1.45$ & - \\
\hline
\end{tabular}

Table 3: Accuracy, in degrees, versus the number of patches used for our illuminant estimation method on the multispectral outdoor image database captured under both the visible and near-infrared spectra compared to Finlayson and Schaefer's method (F \& S) and Sunshine et al.'s approach.

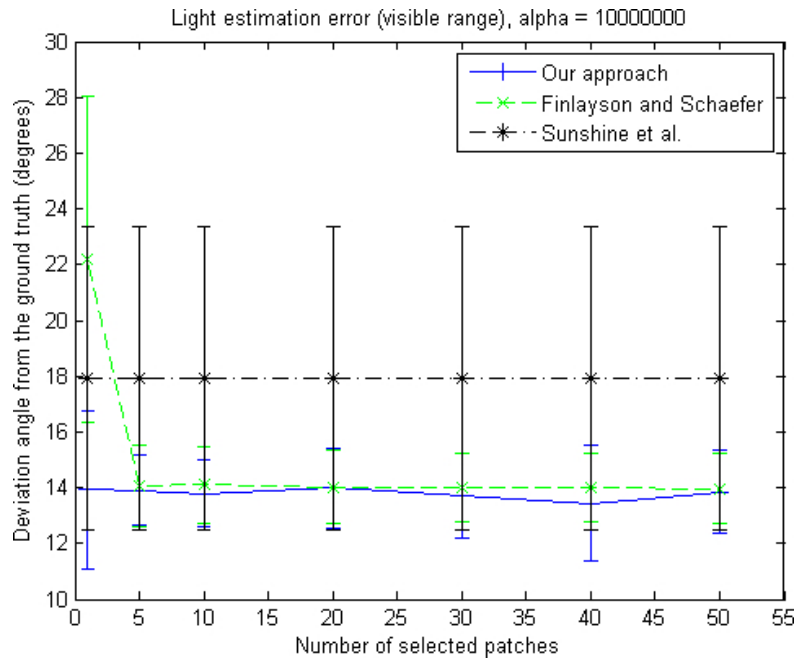

(a) Visible spectrum

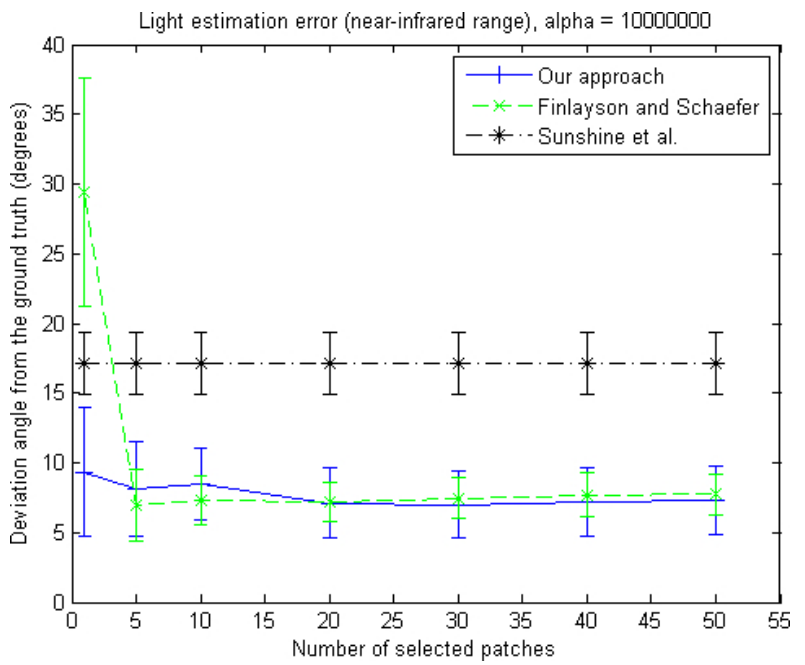

(b) Near-infrared spectrum

Figure 4: Accuracy versus the number of patches used for our illuminant estimation method on the multispectral outdoor image database, in degrees, compared to Finlayson and Schaefer's method (F \& S) and Sunshine et al.'s method. The results for both the visible (left) and near-infrared ranges (right) are shown. 

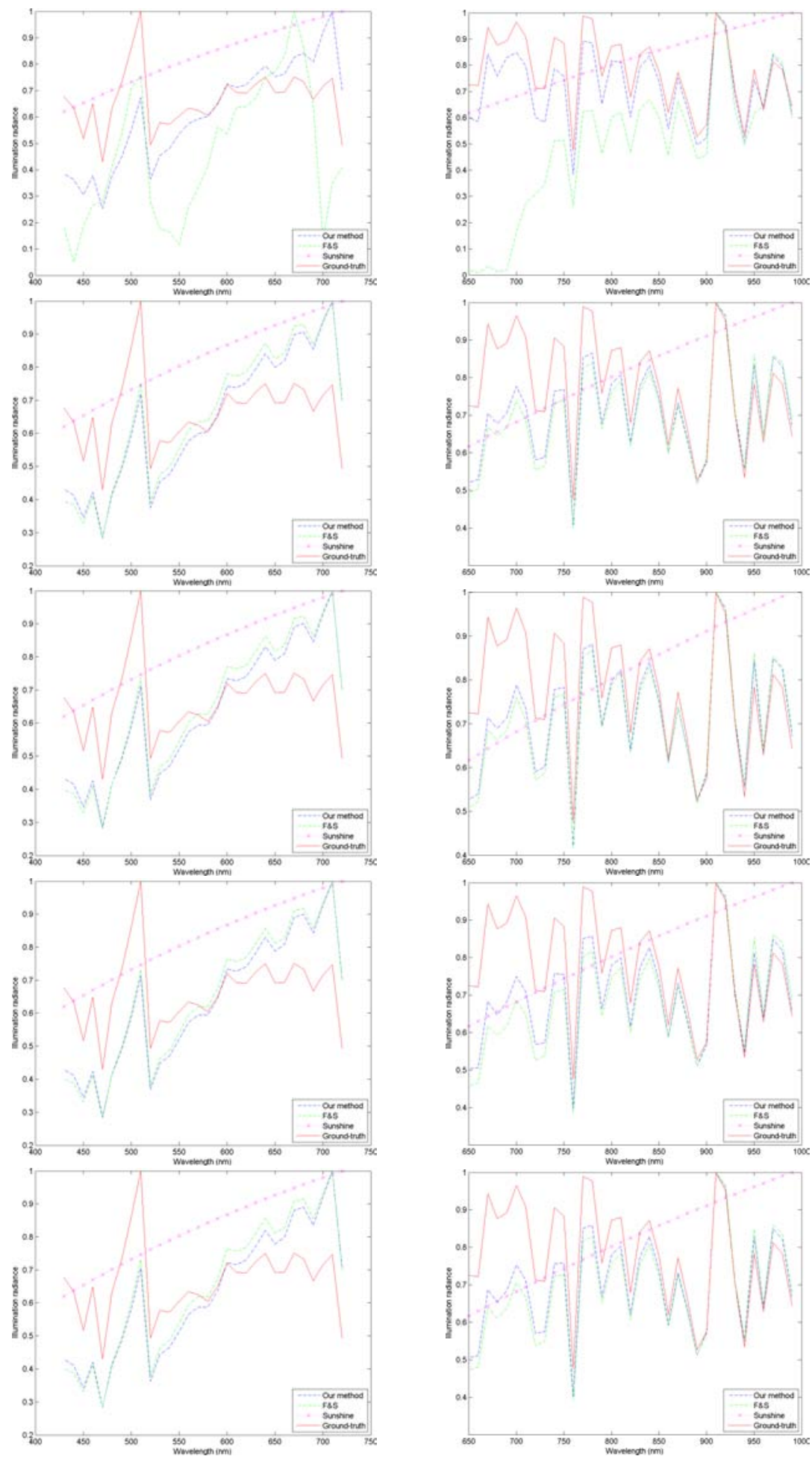

Figure 5: Ground truth illuminant spectra and those estimated by our method and the alternatives from the image of an outdoor scene. Here we show the estimated illuminant spectra in both the visible (left column) and the near-infrared (right column) ranges. From top to bottom: the spectra estimated using 1, 10, 20, 30 and 40 patches from the image. 
image database is lower than that for the face database due to a wider variation of spectral radiance across the scene. These trends are visually demonstrated using a number of sample plots of the estimated spectra of natural sunlight, as shown in Figure 5.

\subsubsection{Trichromatic Light Recovery}

Next, we turn our attention to the utility of our parameter recovery method for the purpose of illuminant colour estimation from trichromatic images. To this end, we generate RGB imagery from the multispectral face and outdoor databases mentioned previously. These are synthesized by simulating a number of trichromatic spectral responses, including the CIE-1932 colour matching functions [15] and the camera sensors for a Nikon D70 and a Canon 10D. Furthermore, we apply our method and the alternatives to the Mondrian and specular image datasets as described by Barnard et al [4]. We also compare the performance of our algorithm with several colour constancy approaches described in [3] by the same authors.

To illustrate the effect of varying the value of $\alpha$, we perform experiments with $\alpha=10000$ and $\alpha=100$. In Table 4 and Figure 6 we show results for the light estimation accuracy on the RGB face images with $\alpha=10000$ and a patch size of $20 \times 20$ pixels. Our method outperforms the alternatives in terms of estimation accuracy and stability when the number of patches (or the number of available intrinsic colours in the scene) is 5 or less. Another general trend is that our method and Finlayson and Schaefer's [21] one improve their accuracy as the number of selected patches increases. However, this improvement is marginal for our method when we use 20 or more patches. Meanwhile, the method of Finlayson and Schaefer [21] tends to achieve a closer estimate to the groundtruth when it is applied on a sufficiently large number of patches from the images synthesized for the Canon 10D and Nikon D70 sensors. Interestingly, for images simulated for the colour matching functions, which emulate the human visual perception, our method achieves a similar accuracy to Finlayson and Schaefer's [21] across all the number of selected patches, while being more stable (with lower variance of angular error). In all our experiments, the approach of Sunshine et. al [53] is the one that delivers the worst performance.

Table 5 and Figure 7 show the accuracy of the illuminant colour estimation on the outdoor RGB image database, with $\alpha=100$ and a patch size of $20 \times 20$ pixels. The major trend of these statistics is that our method achieves an accuracy significantly higher than those achieved by the others. The difference in performance is in the order of several standard deviations of the angular 


\begin{tabular}{|c|c|c|c|c|c|c|c|c|}
\hline \multicolumn{2}{|c|}{ Number of patches } & 1 & 5 & 10 & 20 & 30 & 40 & 50 \\
\hline \multirow{3}{*}{$\sum_{U}^{L}$} & Our method & $3.84 \pm 2.86$ & $4.18 \pm 2.89$ & $4.16 \pm 2.79$ & $3.99 \pm 2.61$ & $3.94 \pm 2.58$ & $3.84 \pm 2.54$ & $3.83 \pm 2.55$ \\
\hline & $F \& S$ & $24.97 \pm 10.29$ & $5.58 \pm 4.68$ & $4.50 \pm 3.72$ & $3.88 \pm 3.15$ & $3.70 \pm 3.04$ & $3.66 \pm 3.02$ & $3.59 \pm 2.99$ \\
\hline & Sunshine & $6.09 \pm 2.60$ & $6.09 \pm 2.60$ & $6.09 \pm 2.60$ & $6.09 \pm 2.60$ & $6.09 \pm 2.60$ & $6.09 \pm 2.60$ & $6.09 \pm 2.60$ \\
\hline \multirow{3}{*}{ 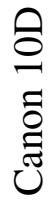 } & Our method & $3.91 \pm 2.87$ & $4.34 \pm 3.01$ & $4.15 \pm 2.72$ & $4.09 \pm 2.52$ & $3.98 \pm 2.45$ & $3.90 \pm 2.36$ & $3.86 \pm 2.34$ \\
\hline & $F \& S$ & $25.37 \pm 10.81$ & $3.72 \pm 3.00$ & $3.22 \pm 2.17$ & $2.82 \pm 1.70$ & $2.62 \pm 1.46$ & $2.54 \pm 1.40$ & $2.46 \pm 1.35$ \\
\hline & Sunshine & $5.84 \pm 2.06$ & $5.84 \pm 2.06$ & $5.84 \pm 2.06$ & $5.84 \pm 2.06$ & $5.84 \pm 2.06$ & $5.84 \pm 2.06$ & $5.84 \pm 2.06$ \\
\hline \multirow{3}{*}{$\begin{array}{l}0 \\
0 \\
\frac{1}{0} \\
\frac{1}{Z}\end{array}$} & Our method & $3.88 \pm 2.82$ & $4.26 \pm 2.88$ & $4.17 \pm 2.88$ & $3.91 \pm 2.49$ & $3.93 \pm 2.46$ & $3.85 \pm 2.41$ & $3.86 \pm 2.40$ \\
\hline & $F \& S$ & $25.67 \pm 10.83$ & $4.26 \pm 3.94$ & $3.22 \pm 2.30$ & $2.74 \pm 1.67$ & $2.59 \pm 1.50$ & $2.48 \pm 1.37$ & $2.43 \pm 1.31$ \\
\hline & Sunshine & $5.84 \pm 2.06$ & $5.84 \pm 2.06$ & $5.84 \pm 2.06$ & $5.84 \pm 2.06$ & $5.84 \pm 2.06$ & $5.84 \pm 2.06$ & $5.84 \pm 2.06$ \\
\hline
\end{tabular}

Table 4: Accuracy of our illuminant estimation method with $\alpha=10000$ on the synthesized RGB face image database, in degrees, compared to Finlayson and Schaefer's method (F \& S) and Sunshine et al.'s approach. We show the results on RGB face images synthesized from the multispectral face imagery for the Stiles and Burch's colour matching functions (rows 2-4), the spectral sensitivity response functions of a Canon 10D (rows 5-7) and a Nikon D70 camera (rows 8-10).

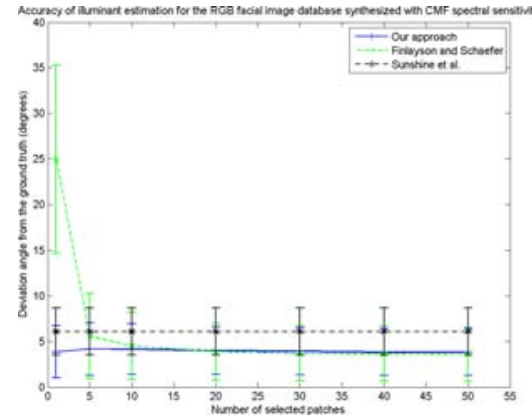

(a) $\mathrm{CMF}$

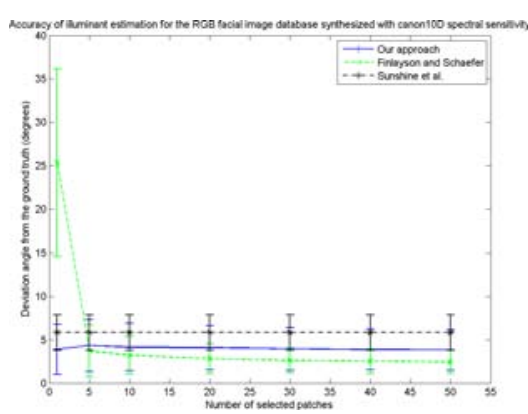

(b) Canon 10D

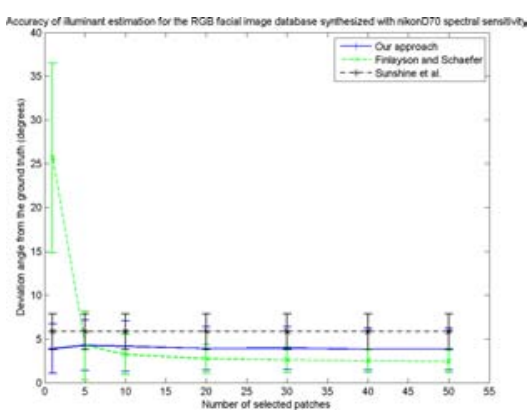

(c) Nikon D70

Figure 6: Accuracy versus number of image patches for our illuminant estimation method with $\alpha=10000$ on the RGB face images synthesized from the multi-spectral face imagery, in degrees, compared to Finlayson and Schaefer's method (F \& S) and Sunshine et al.'s approach. From left to right: Results for simulated RGB images as captured with (a) Stiles and Burch's colour matching functions (b) a Canon 10D camera sensor (c) a Nikon D70 camera sensor.

error. While the performance of our method is slightly degraded as the number of patch increases (above 20), the stability of the estimate remains constant for Canon 10D and Nikon D70 images, and even improves for the images simulated for the CIE 1932 standard colour matching functions. 


\begin{tabular}{|c|c|c|c|c|c|c|c|c|}
\hline \multicolumn{2}{|c|}{ Number of patches } & 1 & 5 & 10 & 20 & 30 & 40 & 50 \\
\hline \multirow{3}{*}{$\sum_{U}^{L}$} & Our method & $0.46 \pm 0.38$ & $0.50 \pm 0.36$ & $0.97 \pm 1.60$ & $1.32 \pm 2.02$ & $1.40 \pm 1.72$ & $2.18 \pm 2.23$ & $2.34 \pm 2.27$ \\
\hline & $\mathrm{F} \& \mathrm{~S}$ & $23.93 \pm 6.66$ & $10.74 \pm 2.27$ & $11.23 \pm 2.03$ & $11.28 \pm 0.98$ & $11.04 \pm 0.91$ & $10.95 \pm 1.00$ & $10.99 \pm 0.89$ \\
\hline & Sunshine & $11.35 \pm 1.46$ & $11.35 \pm 1.46$ & $11.35 \pm 1.46$ & $11.35 \pm 1.46$ & $11.35 \pm 1.46$ & $11.35 \pm 1.46$ & $11.35 \pm 1.46$ \\
\hline \multirow{3}{*}{ 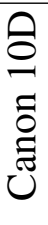 } & Our method & $0.94 \pm 1.32$ & $0.82 \pm 1.22$ & $1.08 \pm 1.56$ & $1.08 \pm 1.63$ & $1.67 \pm 2.06$ & $1.76 \pm 2.08$ & $2.22 \pm 2.37$ \\
\hline & $\mathrm{F} \& \mathrm{~S}$ & $22.41 \pm 9.68$ & $9.33 \pm 2.22$ & $9.42 \pm 2.06$ & $9.68 \pm 1.45$ & $9.66 \pm 1.14$ & $9.73 \pm 1.03$ & $9.63 \pm 0.99$ \\
\hline & Sunshine & $10.33 \pm 1.40$ & $10.33 \pm 1.40$ & $10.33 \pm 1.40$ & $10.33 \pm 1.40$ & $10.33 \pm 1.40$ & $10.33 \pm 1.40$ & $10.33 \pm 1.40$ \\
\hline \multirow{3}{*}{ 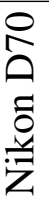 } & Our method & $0.78 \pm 1.42$ & $0.66 \pm 0.66$ & $0.90 \pm 1.02$ & $1.60 \pm 2.17$ & $1.99 \pm 2.63$ & $2.24 \pm 2.57$ & $2.70 \pm 2.82$ \\
\hline & $\mathrm{F} \& \mathrm{~S}$ & $24.98 \pm 8.74$ & $9.98 \pm 1.59$ & $10.13 \pm 2.98$ & $9.88 \pm 1.22$ & $9.88 \pm 1.14$ & $9.65 \pm 1.20$ & $9.55 \pm 1.00$ \\
\hline & Sunshine & $10.33 \pm 1.40$ & $10.33 \pm 1.40$ & $10.33 \pm 1.40$ & $10.33 \pm 1.40$ & $10.33 \pm 1.40$ & $10.33 \pm 1.40$ & $10.33 \pm 1.40$ \\
\hline
\end{tabular}

Table 5: Accuracy of our illuminant estimation method with $\alpha=100$ on the synthesized RGB outdoor image database, in degrees, compared to Finlayson \& Schaefer's method (F \& S) and Sunshine et al.'s approach. We show the results on RGB outdoor images synthesized from the multispectral outdoor imagery for the Stiles and Burch's colour matching functions (rows 2-4), the spectral sensitivity response functions of a Canon 10D (rows 5-7) and a Nikon D70 camera (rows 8-10).

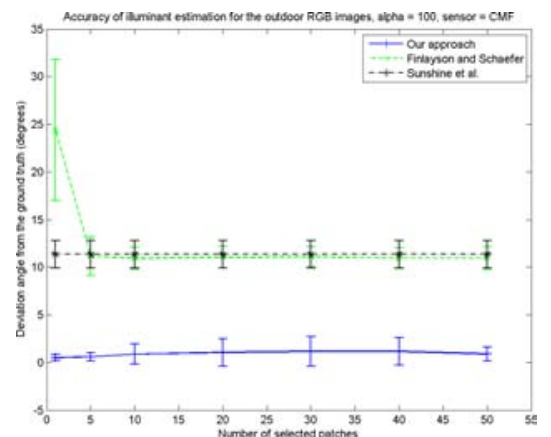

(a) $\mathrm{CMF}$

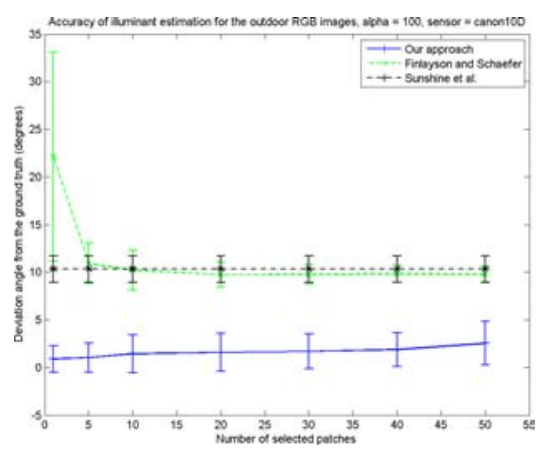

(b) Canon 10D

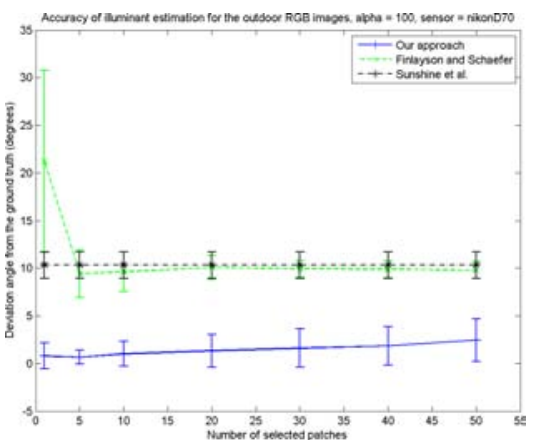

(c) Nikon D70

Figure 7: The accuracy of our illumant estimation method with $\alpha=100$ on the RGB outdoor images synthesized from the multi-spectral image database, in degrees, compared to Finlayson \& Schaefer's method (F \& S) and Sunshine et al.'s method, versus the number of patches used. From left to right are the results for simulated RGB images as captured with (a) Stiles and Burch's colour matching functions [15] (b) a Canon 10D camera sensor and (c) a Nikon D70 camera sensor. 


\begin{tabular}{|c|c|c|c|c|c|c|}
\hline \multirow{2}{*}{$\begin{array}{c}\text { No. } \\
\text { patches }\end{array}$} & \multicolumn{3}{|c|}{ Standard dynamic range (8 bits) } & \multicolumn{3}{c|}{ Extended dynamic range (16 bits) } \\
\cline { 2 - 7 } & Our method & F \& S & Sunshine & Our method & F \& S & Sunshine \\
\hline 1 & $8.45 \pm 6.97$ & $25.58 \pm 13.72$ & $12.92 \pm 9.23$ & $8.78 \pm 7.86$ & $24.70 \pm 13.61$ & $12.45 \pm 8.67$ \\
\hline 5 & $7.78 \pm 6.23$ & $9.88 \pm 10.71$ & - & $8.63 \pm 7.75$ & $12.85 \pm 11.90$ & - \\
\hline 10 & $7.78 \pm 6.46$ & $10.06 \pm 10.89$ & - & $8.18 \pm 7.18$ & $11.73 \pm 11.00$ & - \\
\hline 20 & $7.77 \pm 6.69$ & $9.41 \pm 9.70$ & - & $7.88 \pm 7.04$ & $10.58 \pm 9.81$ & - \\
\hline 30 & $7.75 \pm 6.90$ & $9.29 \pm 9.76$ & - & $7.92 \pm 7.03$ & $10.30 \pm 9.57$ & - \\
\hline 40 & $7.82 \pm 6.81$ & $9.12 \pm 9.41$ & - & $7.81 \pm 7.03$ & $10.18 \pm 9.54$ & - \\
\hline 50 & $7.88 \pm 6.77$ & $9.25 \pm 9.54$ & - & $7.75 \pm 6.84$ & $10.16 \pm 9.46$ & - \\
\hline
\end{tabular}

Table 6: Accuracy of our illuminant estimation method with $\alpha=1000$ on the Mondrian and specular datasets reported in [4], in degrees, compared to Finlayson and Schaefer's method (F \& S) and Sunshine et al.'s approach. The accuracy is measured in degrees.

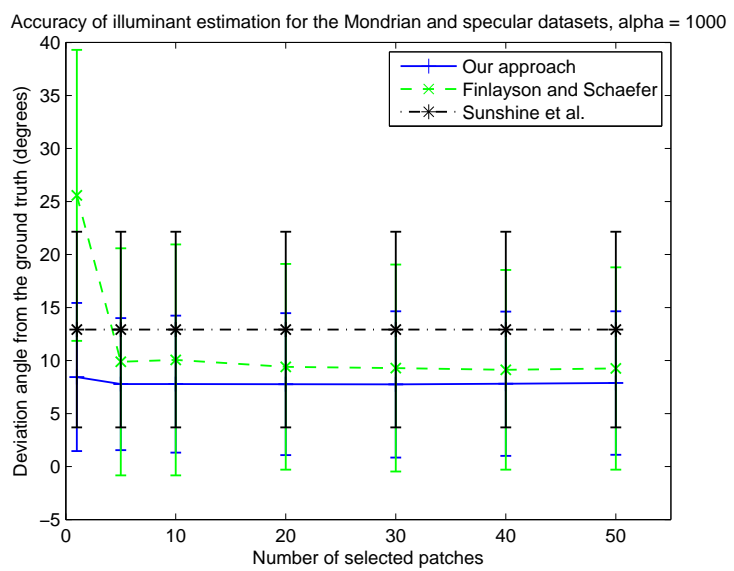

(a) Standard dynamic range (8 bits)

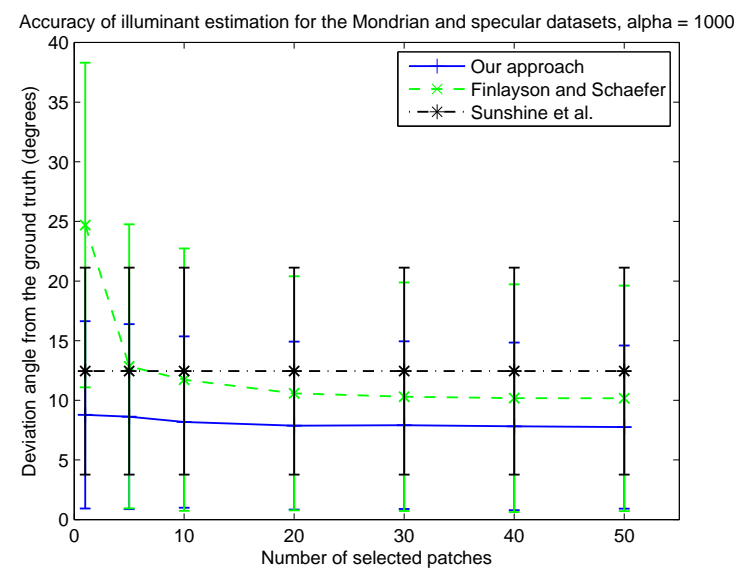

(b) Extended dynamic range (16 bits)

Figure 8: Accuracy of our illuminant estimation method with $\alpha=1000$ for the Mondrian and specular datasets with 8-bit and 16-bit dynamic ranges, as reported in [4]. Our method is compared to that of Finlayson and Schaefer (F \& S) and Sunshine et al..

It is also important to notice that our method performs better on the imagery synthetised using the CIE 1932 standard. This is consistent with the results reported for the RGB face images. Overall, our method appears to outperform the alternatives when applied to cluttered scenes with a high variation in colour and texture.

Next, we turn our attention to the illuminant estimation accuracy on the Mondrian and specular image datasets reported in [4]. To account for the level of texture density in some of the images, 
we choose a patch size of $10 \times 10$ pixels, which is small enough so that the assumption of uniform albedo across each patch still holds. In this experiment, a patch is regarded to be of homogeneous reflectance if $75 \%$ or more of the patch pixels deviate by less than 1 degree from their projection on the dichromatic plane of the patch. We also enforce a criterion that precludes the selection of highly contrasting patches which contain more than one material or saturated highlight pixels. Specifically, we rank patches in an image by their contrast levels and select the most contrasting ones, excluding those in the top $10 \%$ in each image of the Mondriant dataset. For the specular dataset, we exclude the top $30 \%$ percent of the patches in each image to accommodate for a higher level of colour saturation.

In Table 6 and Figure 8, we show the accuracy when 1, 5, 10, 20, 30, 40 and 50 patches are used. Our results are consistent with previous experiments, which shows that our method outperforms the alternatives on both the 8-bit and 16-bit datasets. This is reflected not only by a lower mean of angular error yielded by our method, but also a lower standard deviation of its performance. In addition, our method delivers a variance of angular error which is almost constant with 5 or more selected patches. Further, the performance of our method improves slightly by increasing the number of selected patches up to 30 for the 8-bit dynamic range and up to 50 for the 16 -bit dynamic range. To some extent, our estimator appears to be insensitive to the dynamic range of the input image. This shows that our method is more stable and robust to variations in the scene reflectance.

In comparison to the benchmark methods reported by Barnard et al. [3], our method ranks second, just below the gamut mapping methods presented in [23]. The methods in [23] deliver an accuracy between $5.6-7.1$ degrees for the 16-bit images and $6.3-8.3$ degrees for the 8 -bit images, as shown in Table II in [3]. However, these results were reported for imagery that had already undergone several processing steps including segmentation, scaling and clipping operations. On the other hand, our method does not require any preprocessing and, moreover, it is capable of recovering all the dichromatic-model parameters being applicable to hyperspectral imagery with trichomatic as a particular case.

\subsubsection{Noise perturbation analysis}

In this section, we examine the robustness of our algorithm to added image noise. To do this, we perturb the multispectral face image database with various levels of additive Gaussian noise. The 


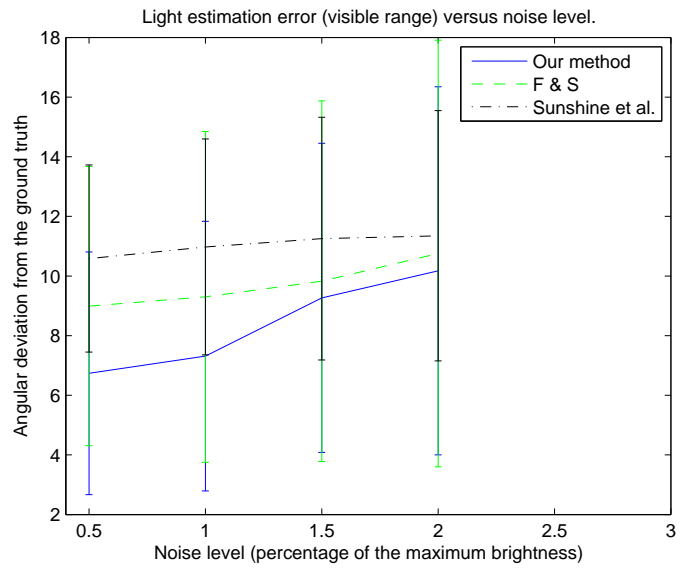

(a) Visible spectrum

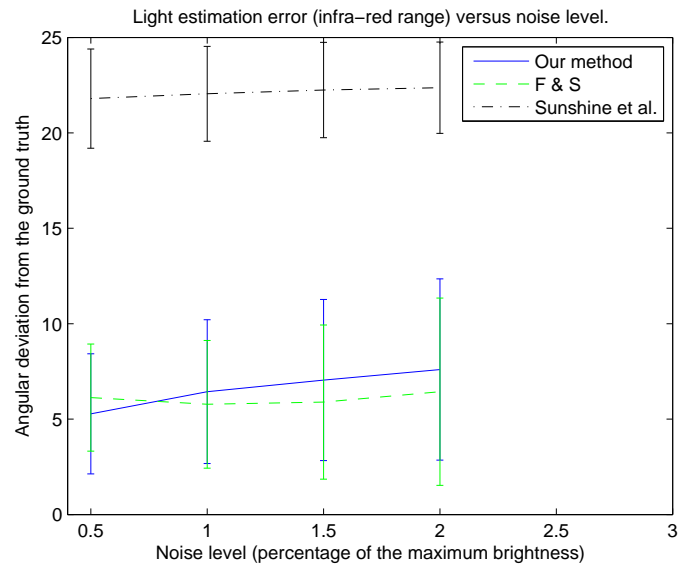

(b) Infra-red spectrum

Figure 9: Accuracy of the estimated illuminant spectra versus the standard deviation of Gaussian noise. The vertical axis shows the angular deviation of the estimated spectra from the corresponding ground-truth, while the horizontal axis shows the standard deviation of Gaussian noise as the percentage of maximum brightness of the imagery. The performance for the visible spectrum is shown in the left-hand image, while that corresponding to the infra-red spectrum is shown on the right-hand panel.

noise has an increasing standard deviation between 0.5 and $2 \%$ of the maximum image brightness, with increments of $0.5 \%$. In Figure 9, we plot the performance of our algorithm and the alternatives across various levels of noise, in the visible (left-hand panel) and infra-red (right-hand panel) ranges. For our algorithm and Finlayson and Schaefer's one [21], we employ all the homogenous patches recovered from the images. The regularisation weight for our method is $\alpha=100000$. As shown in Figure 9, our method achieves a lower mean and standard deviation of the angular error than the other two in the visible spectrum. Moreover, in the infra-red spectrum, our method greatly outperforms Sunshine et al.'s [53] by more than two standard deviations of the angular error in the recovered illuminant spectra.

On the degradation in performance with an increasing level of noise, the Sunshine et al.'s method is most stable because it considers the upper bound of all the radiance spectra in an image, which is least affected by the level of Gaussian noise. However, it is the least accurate method of the three alternatives. Ours and Finlayson \& Schaefer's appear to degrade linearly with the level of noise, although the latter one degrades at a slower rate than our method. This can be explained by the fact that Finlayson \& Schaefer's method relies on an eigenvalue decomposition, which is equivalent to our method with a zero-regularisation term. Albeit obtaining a more robust solution for the illuminant, their method does not take surface shading and highlights into account. Not 
only can our approach estimate the illuminant spectrum, it is also capable of computing all the dichromatic parameters, while maintaining a reasonable estimation using regularisation.

\subsection{Skin Recognition and Material Clustering}

We now turn our attention to the illumination invariance of the spectral reflectance recovered by our algorithm and its applications to recognition tasks. Firstly, we focus on using the spectral image reflectance extracted according to Section 2.4 for skin recognition. This task can be viewed as a classification problem where the skin and non skin spectra comprise positive and negative classes, respectively. In this manner, we can assert the robustness and consistency of both the illuminant spectrum and surface reflectance recovered by our algorithm at training time, and those yielded by the method for skin recognition at the testing phase.

In this experiment, we compare the skin recognition performance yielded using the reflectance spectra recovered by our method as the feature for classification to those results yielded by the classifier using a number of alternative features. To this end, we present the results for two variants of our recovered reflectance, both estimated by the procedure described in Section 2.4. For the first variant, the ground-truth illuminant spectrum is supplied as input. For the second one, we use the estimated illuminant spectra obtained by the experiments in Section 5.1.1. By comparing the performance in these two cases, we can assess the robustness of the recovered reflectance when the estimated illuminant spectra is used as compared to the ground-truth. In addition, we also compare these variants with a number of alternatives. The first of these is the spectral reflectance obtained by normalising the raw image radiance spectra by the measured ground-truth illuminant. The second case is where the classifier is applied to the raw radiance spectra. Lastly, we use the principal components resulting from performing subspace projection via Linear Discriminant Analysis (LDA) on the original radiance spectra.

This experiment is performed on the face image database captured in the visible range described earlier. To obtain a training data-set, we select skin and non skin regions from an image captured under a light source placed in a high-oblique position in front of the subject. On average, there are 856 skin pixels and 7796 non-skin pixels selected from several regions in each image as training data. Subsequently, each of the features described above is extracted from each training set and used as input to a Support Vector Machine (SVM) classifier [13] with a Radial Basis Function (RBF) kernel. In addition, the parameters are selected using 5-fold cross validation at training 
time. To classify skin versus non skin pixels, the resulting SVM model is applied to the test images of the same subject. The test images, each with a size of $340 \times 400$ pixels, have been acquired under other illuminant conditions.

In Figure 10 we present the skin segmentation maps obtained using the input features described above. The top row shows the training images of a number of sample subjects, with skin training regions enclosed in red rectangular boundaries and non-skin training areas enclosed in blue rectangles. The second row, from top-to-bottom, shows the test images for the subjects in the top row. Note that the illuminant directions and power spectra in the two rows differ, as can be observed in the shading and shadows. In fact, the training images are illuminated by the light source placed in a high-oblique position in front of the subjects whereas the test images are illuminated by a frontal light source with the same direction as the viewing direction. The bottom five rows are the skin probability maps yielded by the SMVs trained using the features described above. In the figure, lighter pixels are classified as being more likely to be skin. The third and fourth rows correspond to the variants of our recovered reflectance, with ground-truth illuminant and estimated illuminant spectra supplied as input, respectively. The fifth, sixth and seventh rows correspond to the reflectance obtained by normalising the image radiance by the ground-truth illuminant spectrum, the raw radiance and the top 20 LDA components of the image radiance spectra, respectively.

From Figure 10, we can conclude that the skin reflectance spectra recovered by our method are, in fact, invariant to illuminant power and direction. This stems from the fact that the reflectance features delivered by our method yield the most visually accurate skin maps. In many cases, nonskin face details such as eyebrows and mouth are correctly distinguished from skin. Furthermore, the results of the two reflectance variants are highly consistent. This is due to the low diference between the estimated illuminant and the ground truth, which deviate typically between 1 and 3 degrees.

On the other hand, the reflectance features used for the results on the fifth row, although being illuminant invariant, still yield falsely classified skin pixels. The poor classification results obtained by these features can be explained by the variation induced by the illuminant spectrum and the surface shading. This is evident at pixels near the face boundary and the highlight positions. This is expected in the fifth row since normalising radiance by illuminant power does not achieve surface shading-independence and disregards the specular components inherent to the dichromatic the model. In contrast, our method achieves the recovery of the reflectance free of geometry and specularity artifacts. Thus it is able to recognise skin pixels at grazing angles and specular spikes. 

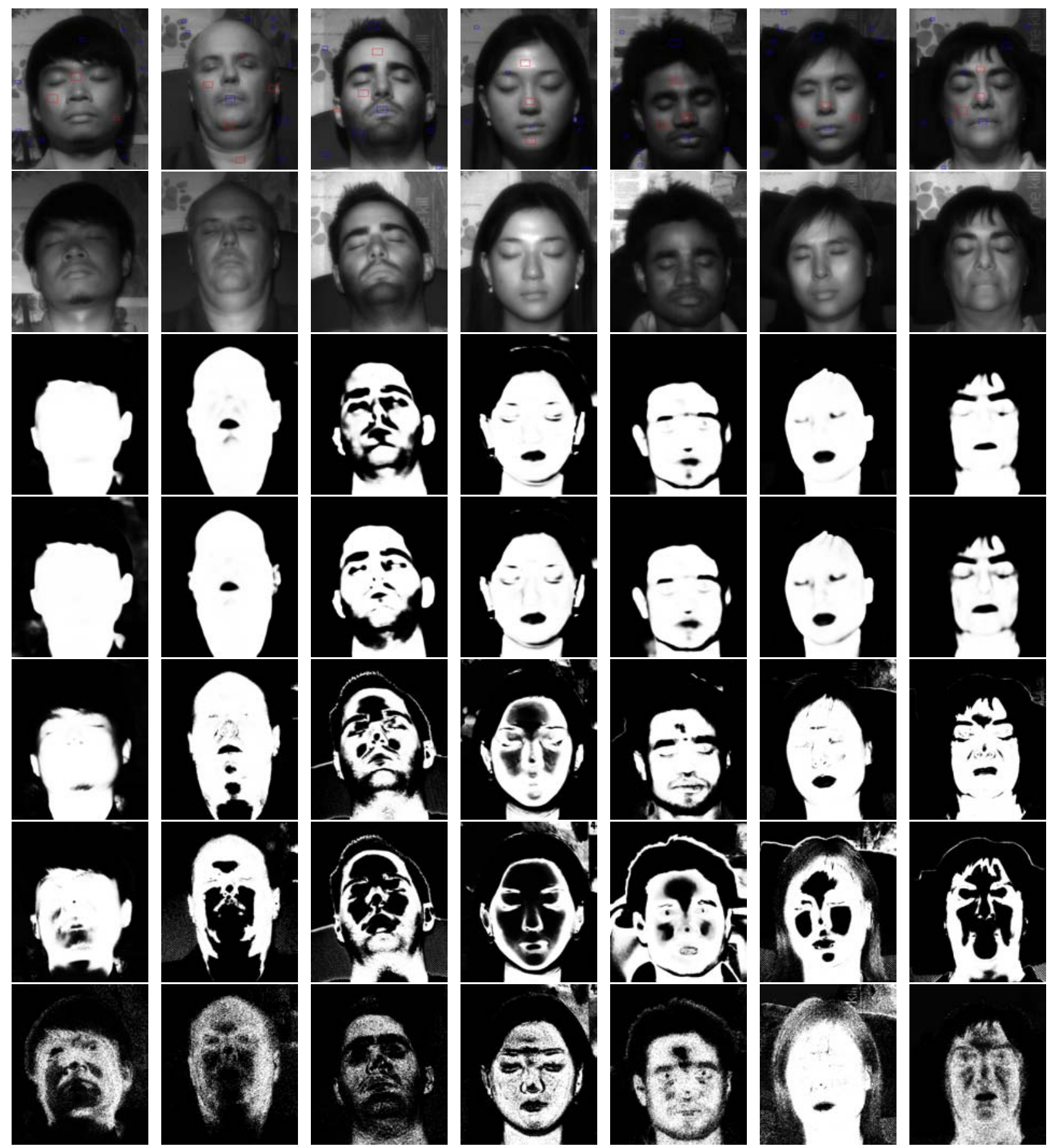

Figure 10: Skin segmentation results. Top row: sample training images for skin recognition, with labelled skin regions (with red borders) and non-skin regions (in blue borders). Second row: the test images of the corresponding subjects, captured under a different illumination direction. The third to last row are the skin probability map obtained using different features. Third row: obtained using the reflectance estimated given the ground-truth illuminant spectrum. Fourth row: yielded using the estimated reflectance after estimating the illuminant spectrum. Fifth row: yielded using the reflectance obtained by normalising radiance spectra by the ground-truth illuminant spectrum. Sixth row: yielded using the raw radiance of the input images. Seventh row: recovered making use of the top 20 LDA components of the raw radiance spectra. 


\begin{tabular}{|c|c|c|c|}
\hline Feature for classification & CDR $(\%)$ & FDR $(\%)$ & CR $(\%)$ \\
\hline Estimated reflectance \& ground-truth light & $\mathbf{8 5 . 1 2} \pm \mathbf{1 3 . 3 6}$ & $\mathbf{5 . 1 0} \pm \mathbf{6 . 3 0}$ & $\mathbf{9 0 . 9 4} \pm \mathbf{6 . 1 2}$ \\
\hline Estimated reflectance \& estimated light & $79.79 \pm 22.58$ & $8.32 \pm 15.78$ & $87.00 \pm 13.63$ \\
\hline Reflectance by illuminant normalisation & $70.63 \pm 16.95$ & $5.50 \pm 5.69$ & $84.75 \pm 8.04$ \\
\hline Raw radiance & $47.27 \pm 20.58$ & $12.48 \pm 11.64$ & $71.23 \pm 9.17$ \\
\hline Top 20 LDA components of raw radiance & $44.83 \pm 31.47$ & $7.14 \pm 11.42$ & $73.62 \pm 11.54$ \\
\hline
\end{tabular}

Table 7: Accuracy of several skin pixel recognition methods, each using a different reflectance-based feature as input for classification.

In addition, normalised raw radiance spectra and their LDA components, as employed for the classification on the sixth and seventh rows are not illuminant invariant. Therefore these cannot cope with the change in illumination between the training and test images. As shown in the last two rows, this results in much more false negatives in skin areas and false positives in other materials as compared to the reflectance features yielded by our method.

In order to provide a quantitative analysis, in Table 7 we show the performance of the above skin segmentation schemes in terms of the classification rate $(C R)$, the correct detection rate $(C D R)$ and false detection rate $(F D R)$. The correct detection rate is the percentage of skin pixels correctly classified. The false detection rate is the percentage of non-skin pixels incorrectly classified. The classification rate is the overall percentage of skin and non-skin pixels classified accurately. The table shows the segmentation accuracy measures over all the visible face images of all the subjects in the dataset illuminated by the frontal light source. The rows of the table correspond to the different skin classification features described earlier. As expected, the reflectance recovered by our method achieves the highest skin recognition rates. This is consistent with the qualitative results above. Furthermore, the overall performance difference between the two reflectance variants based upon our method, i.e. when the estimated and the ground-truth light spectrum are used, is less than $4 \%$. This demonstrates the robustness of our reflectance estimation method to errors in the input illuminant spectrum. As before, the reflectance obtained by normalising radiance by illuminant power performs better than the raw radiance spectra and its LDA components. Again, the radiance feature and its LDA components yield the most false positives and negatives.

Next, we examine the utility of the spectral reflectance recovered by our algorithm for unsupervised material clustering on multispectral images. This experiment can be regarded as an extension 

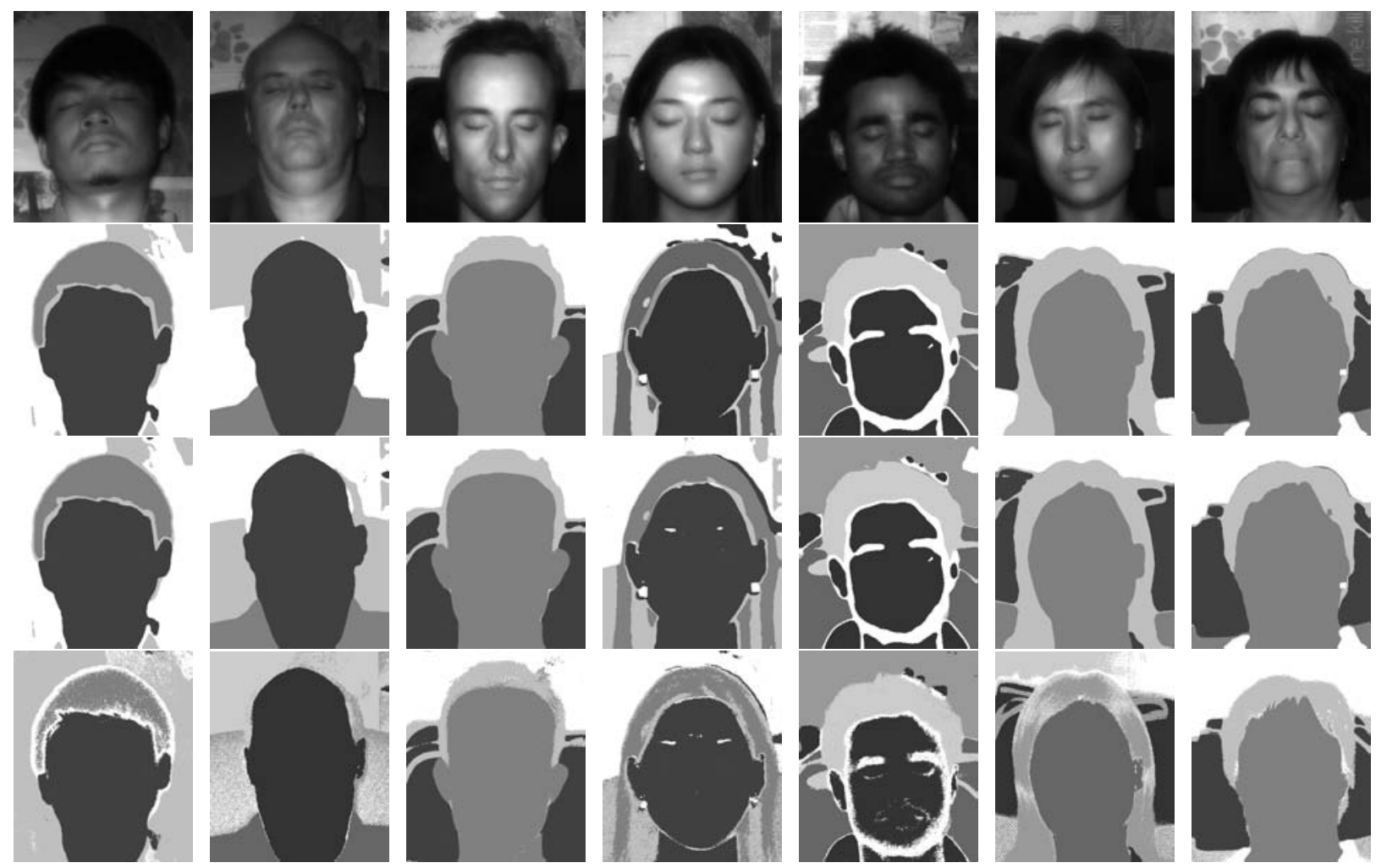

Figure 11: Material clusters, with each material marked by a different shade of gray. Top row: A band of the input images, shown at $670 \mathrm{~nm}$. Second row: material clustering maps, obtained with the clustering feature being the estimated reflectance given the ground-truth illuminant. Third row: material clustering maps resulting from the use of the reflectance feature recovered with an estimated illuminant spectrum as input. Fourth row: material clustering maps, using the reflectance obtained by normalising the input radiance image by the ground-truth illuminant spectrum.

of the skin segmentation application. In addition, it is complementary to skin clustering algorithms using trichromatic features, which has been described elsewhere in the literature [44]. It also compares the clustering accuracy on the estimated reflectance to that on the measured (groundtruth) reflectance. Here, we perform a clustering algorithm based on a deterministic annealing approach [27] on the three reflectance features mentioned in the previous experiment. These features include the estimated reflectance estimated given the ground-truth illuminant spectrum, the estimated reflectance yielded from an estimated illuminant spectrum, and the reflectance obtained by normalising radiance spectra by the ground-truth illuminant spectrum. The clustering algorithm is initialised with a single cluster for all the materials. As the algorithm proceeds, new material clusters are introduced by splitting the existing ones. Thus, the resulting number of clusters is 
data-dependent and does not need to be specified as input.

In Figure 11, we show the clustering maps of the images of several human subjects, with each row corresponding to a reflectance feature. The resulting material clusters are marked with different shades of gray. In fact, there are a high level of similarity between the clutering results yielded by the reflectance features estimated with the estimated illumant spectrum and with the ground truth illuminant spectrum provided as input, as shown in rows 2 and 3. This demonstrates, again, the robustness of our reflectance estimation method to errors in the input illuminant spectrum. In these clustering maps, all the materials are well-separated from each other. Moreover, there are very few misclassified pixels within each cluster. On the faces, the skin pixels are clearly distinguished from the neighbouring regions. Notably, the background regions displaying printed faces, such as that in the third column, are correctly clustered as paper. This result demonstrates that the spectral variation of material reflectance is a better feature for classification than trichromatic colour. Note that using trichromatic imagery, it would have been virtually impossible to set apart materials with the same apparent color, such as real faces from printed ones. In the last row, we use the ground truth (measured) reflectance as feature for the clustering algorithm. Compared to our estimated reflectance, the measured reflectance produces noisier clustering maps, with a substantial number of pixels made of the same material assigned to various clusters. In other words, our reflectance recovery method improves the clustering performance by reducing measurement noise in the raw reflectance spectra.

\subsection{Specularity Removal}

Having estimated the illuminant spectra and surface reflectance, and as mentioned previously, we can employ the procedure in Section 2.4 to separate the diffuse from the specular component in multi-spectral imagery. This is feasible in situations where the spectral reflectance varies slowly within a small spatial neighbourhood of the scene. Note that this assumption is a valid one for many real-world surfaces. Thus, each local neighbourhood can be considered as a smooth homogeneous

surface. As a result, the diffuse component at a location $u$ in patch $P$ is estimated as $\hat{\mathbf{D}}(u)=$ $\mathbf{g}(u)\left(\mathbf{L} \bullet \mathbf{S}_{P}\right)$. The specularity component is given by $\mathbf{k}(u) \mathbf{L}$.

Here, we perform highlight removal on the indoor human face image database presented earlier. As mentioned in previous sections, we commence by estimating the illuminant spectra. We consider a neighbourhood of size $11 \times 11$ around each image pixel and assume that the neighbour- 

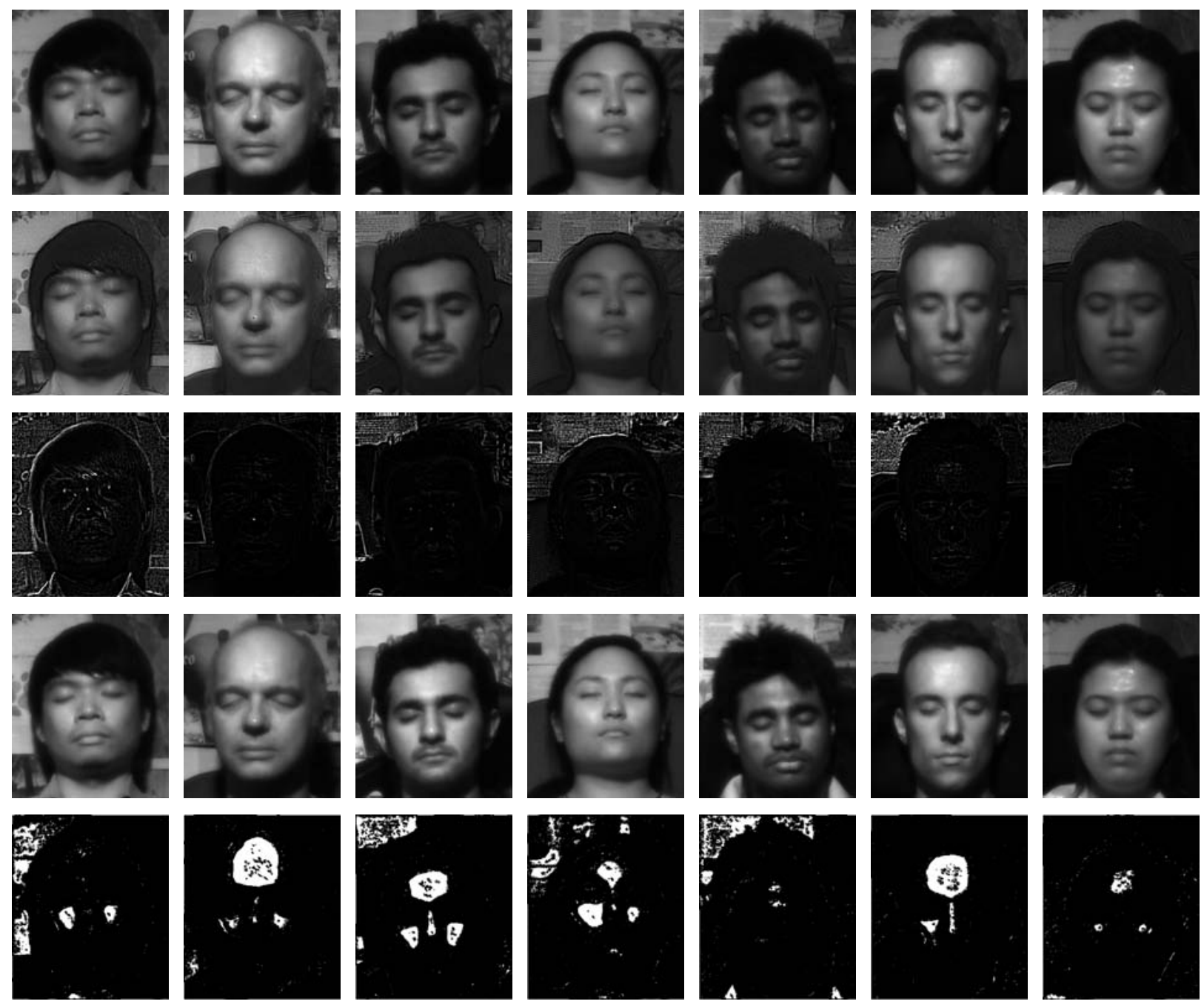

Figure 12: Highlight removal results. First row: original images captured at $670 \mathrm{~nm}$. Second row: the corresponding shading maps produced by our method. Third row: the corresponding specularity images produced by our method. Fourth row: the shading maps produced by Ragheb and Hancock's method [46]. Fifth row: the specularity maps produced by Ragheb and Hancock's method [46].

hood has a common reflectance. As discussed in Section 2.4, a practical enforcement of smooth variation of the shading factor entails reprojecting the pixel radiance onto the subspace spanned by the illuminant spectrum and the diffuse radiance spectrum vectors. In this experiment, we employ a projection that minimises the $L_{2}$-norm of the distance between pixel radiance and this two-dimensional subspace.

Figure 12 shows the resulting shading and specularity coefficients estimated for a number of sample face images in the multispectral dataset. The top row shows the input images illuminated from a high oblique light direction. The second and third rows show the shading and specular 
coefficients, respectively, as yielded from our estimation method. The last two rows show the same results as produced by the alternative highlight removal method in [46]. The alternative uses a probabilistic framework based upon the statistics arising from Lambertian reflectance in diffuse illumination. Note that the alternative is only applicable to a single-band greyscale image compliant with the Lambertian model. Thus, to compare the two methods, we apply the alternative to the brightest band in each of the input images. Also, in order to comply with the assumptions in [46] regarding collinearity of the light and viewer directions, we have applied the alternative method to face images where the camera and the illuminant directions are linearly dependent.

As observed from Figure 12, our method is successful at detecting and separating the specular from the diffuse component at typical highlight locations, such as noses, eyelids and foreheads. In addition, our method produces smooth matte diffuse images that capture the variation in the geometry of faces. Note that our method does not require the illumination direction a priori. On the other hand, Ragheb and Hancock's method [46] assumes the collinearity of the illumination and viewing directions. Therefore, it cannot cope with the application setting shown for our method, where the light source is placed at a high-oblique position with respect to the camera. As expected, the alternative tends to miss important highlight points and generates false specular spikes.

Since our method makes the uniform albedo assumption on input surface patches, it tends to generate highlights in highly textured areas and along material boundaries. However, note that finescale relief texture of rough and highly textured areas may cause specularity that is only detected by elaborate measurements, as discussed in the work of Wang and Dana [58]. Since the background of the multispectral images in Figure 12 may be viewed as textured regions in the images, it may give rise to the highlights detected by our method, as shown in the third row of Figure 12.

Now we turn our attention to the application of our method to specularity detection and removal on trichromatic images. We compare the performance of our method with another highlight removal method [40] which employs a partial differential equation to erode the specularity at each pixel.

In Figure 13, we compare our method with the highlight removal method by Mallick et al. [40]. As before, our method performs better on smooth, homogeneous surfaces than on textured areas, such as those in the second and third rows. On the smooth surfaces, most of the specular spikes are detected by our method, although there may be false specularities along the occlusion boundaries and material boundaries. On the other hand, the alternative produces smoother diffuse components, which are more intuitively correct. In addition, it detects more specular pixels on smooth surfaces. 

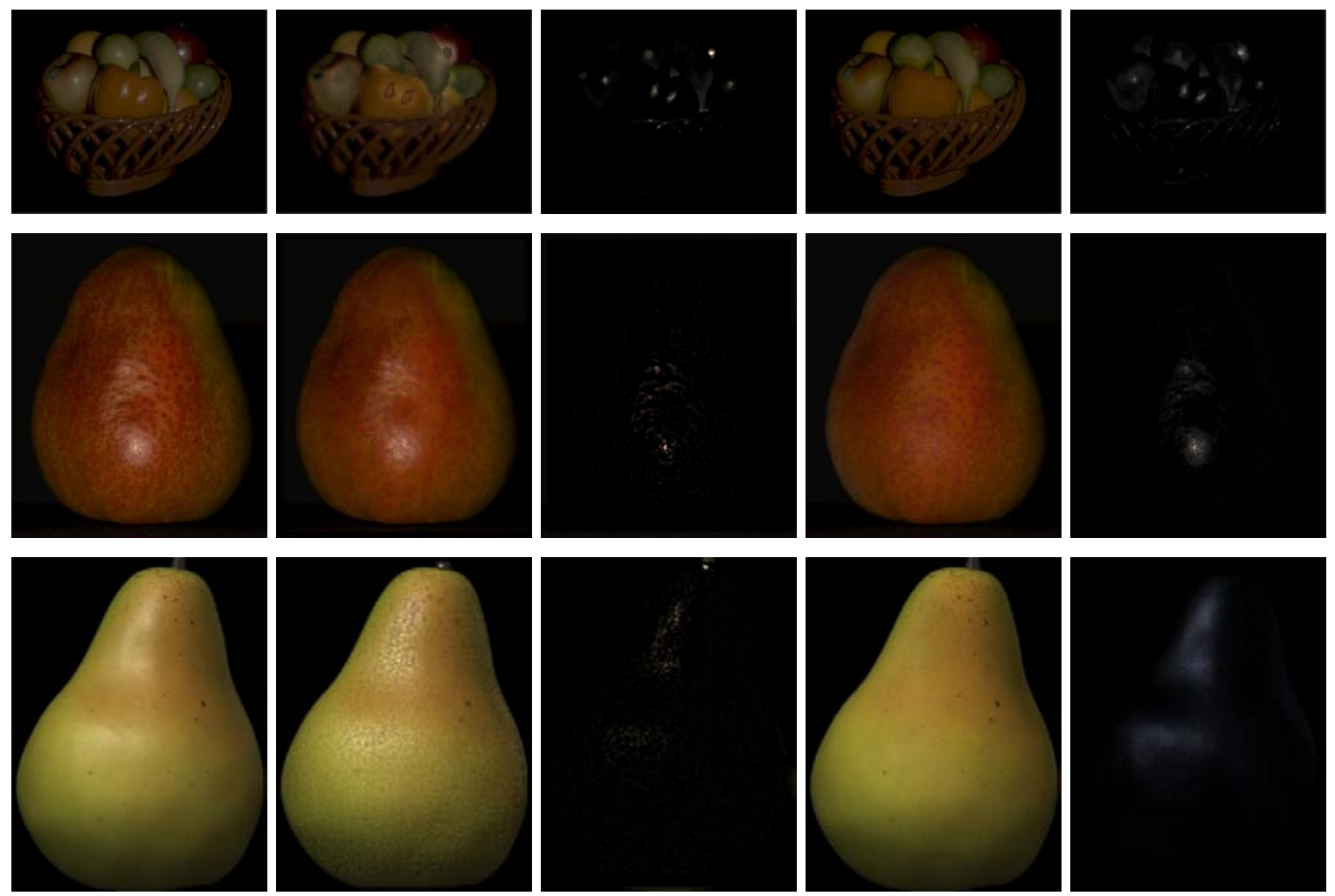

Figure 13: A comparison between the highlight removal results of our method and that by Mallick et al. [40]. First column: the input image. Second and third columns: the diffuse and specular components resulting from our method. Fourth and fifth columns: the diffuse and specular components yielded by Mallick et al.'s method.

As can be seen in the figure, our method is able to detect the expected specular spikes, as shown in the third column. Note that our method may introduce undesirable specularities along edges. This can be observed in the specularity maps in the third row. This is because patches at these locations are not of uniform albedo. Notably, the specularity map in the second row shows the underlying texture variation of the pear, which may be the cause of specularity being scattered over the fruit skin. In the second column, we show the diffuse component recovered by our method, where the diffuse colours at specular points are approximated from the neighbouring non-specular pixels. 


\section{Conclusions}

In this paper, we have presented an optimisation approach so as to recover a solution to the dichromatic model for purposes of photometric invariance from a single multispectral image. The recovery process is based upon optimising a cost function that imposes a smoothness constraint on dichromatic surfaces with uniform reflectance. The method serves several purposes, including scene illuminant estimation, reflectance-based recognition and clustering, and specularity removal. Departing from the dichromatic model, we have presented a cost function which can be optimised using a coordinate descent approach applied to automatically selected surface patches. We have also elaborated upon the use of a number of regularisation strategies so as to enforce smoothness constraints upon the cost function. The method is, hence, quite general in nature, and it is not limited to hyperspectral imagery and is applicable to trichromatic imagery in a straightforward manner. We have shown experiments on real-world imagery where, firstly, the illuminant spectra resulting from our method are shown to outperform those delivered by the alternative methods, especially with a small number of selected homogeneous surface patches. Secondly, we have shown how the reflectance spectra recovered by our method can used for skin recognition and material clustering purposes. Finally, we have shown how specularities can be removed from surfaces to facilitate further computer vision tasks.

\section{References}

[1] Angelopoulou, E. Objective colour from multispectral imaging. In European Conf. on Computer Vision (2000), pp. 359-374.

[2] Angelopoulou, E. Specular highlight detection based on the fresnel reflection coefficient. In IEEE International Conference on Computer Vision (Los Alamitos, CA, USA, 2007), IEEE Computer Society, pp. $1-8$.

[3] Barnard, K., Martin, L., Coath, A., And Funt, B. V. A comparison of computational color constancy Algorithms - Part II: Experiments with image data. IEEE Transactions on Image Processing 11, 9 (2002), 985-996.

[4] Barnard, K., Martin, L., Funt, B., And Coath, A. A Data Set for Colour Research. Color Research and Application 27, 3 (2002), 147-151.

[5] Barnard, K., Martin, L., And Funt, B. V. Colour by Correlation in a Three-Dimensional Colour Space. In ECCV '00: Proceedings of the 6th European Conference on Computer Vision-Part I 
(London, UK, 2000), Springer-Verlag, pp. 375-389.

[6] Boyd, S., And Vandenberghe, L. Convex Optimization. Cambridge University Press, 2004.

[7] Brainard, D., AND WANDEll, B. Analysis of the retinex theory of color vision. Journal of Optical Society America A 3 (1986), 1651-1661.

[8] Brainard, D. H., Delahunt, P. B., Freeman, W. T., Kraft, J. M., and Xiao, B. Bayesian model of human color constancy. Journal of Vision 6, 11 (2006), 1267-1281.

[9] Brainard, D. H., AND Freeman, W. T. Bayesian color constancy. Journal of Optical Society America A 14, 7 (1997), 1393-1411.

[10] Brelstaff, G., ANd Blake, A. Detecting specular reflection using lambertian constraints. In Int. Conf. on Comp. Vision (1988), pp. 297-302.

[11] Brooks, M., AND Horn, B. Shape and source from shading. In MIT AI Memo (1985).

[12] Buchsbaum, G. A Spatial Processor Model for Object Color Perception. Journal of The Franklin Institute 310 (1980), 1-26.

[13] Chang, C.-C., AND Lin, C.-J. LIBSVM: a library for Support Vector Machines, 2001.

[14] Chang, J. Y., Lee, K. M., And Lee, S. U. Shape from shading using graph cuts. In Proc. of the Int. Conf. on Image Processing (2003).

[15] CIE. Commission Internationale de l'Éclairage Proceedings, 1931. Cambridge University Press, Cambridge, 1932.

[16] Dror, R. O., Adelson, E. H., ANd Willsky, A. S. Recognition of Surface Reflectance Properties from a Single Image under Unknown Real-World Illumination. In Proc. of the IEEE Workshop on Identifying Objects Across Variations in Lighting (2001).

[17] Ferrie, F., ANd Lagarde, J. Curvature consistency improves local shading analysis. CVGIP: Image Understanding 55, 1 (1992), 95-105.

[18] Finlayson, G., And Hordley, S. Improving Gamut Mapping Color Constancy. IEEE Transactions on Image Processing 9, 10 (2000).

[19] Finlayson, G. D., Hordley, S. D., ANd Hubel, P. M. Color by Correlation: A Simple, Unifying Framework for Color Constancy. IEEE Transactions on Pattern Analysis and Machine Intelligence 23, 11 (2001), 1209-1221.

[20] Finlayson, G. D., Hordley, S. D., And Tastl, I. Gamut constrained illuminant estimation. Int. J. Comput. Vision 67, 1 (2006), 93-109.

[21] Finlayson, G. D., AND Schaefer, G. Convex and Non-convex Illuminant Constraints for Dichromatic Colour Constancy. CVPR 1 (2001), 598-604.

[22] Finlayson, G. D., AND Schaefer, G. Solving for Colour Constancy using a Constrained Dichromatic Reflection Model. International Journal of Computer Vision 42, 3 (2001), 127-144. 
[23] FoRSYTH, D. A. A novel algorithm for color constancy. International Journal of Computer Vision 5, 1 (1990), 5-36.

[24] Healey, G. Estimating spectral reflectance using highlights. Image and Vision Computing 9, 5 (October 1991), 333-337.

[25] Healey, G., and Slater, D. Invariant recognition in hyperspectral images. In IEEE Conf. on Computer Vision and Pattern Recognition (1999), pp. 1438-1043.

[26] Hoaglin, D. C., Mosteller, F., And Tukey, J. W. Understanding Robust and Exploratory Data Analysis. Wiley-Interscience, 2000.

[27] Hofmann, T., And Buhmann, M. Pairwise data clustering by deterministic annealing. IEEE Tansactions on Pattern Analysis and Machine Intelligence 19, 1 (1997), 1-14.

[28] Horn, B. K. P. Robot Vision. MIT Press, Cambridge, Massachusetts, 1986.

[29] Horn, B. K. P., AND BRooKs, M. J. The variational approach to shape from shading. CVGIP 33, 2 (1986), 174-208.

[30] Huber, P. J. Robust Statistics. Wiley-Interscience, 1981.

[31] IKeuChI, K., AND HoRn, B. Numerical shape from shading and occluding boundaries. Artificial Intelligence 17, 1-3 (August 1981), 141-184.

[32] Klinker, G., Shafer, S., And Kanade, T. A Physical Approach to Color Image Understanding. International Journal of Computer Vision 4, 1 (1990), 7-38.

[33] Klinker, G. J., Shafer, S. A., And Kanade, T. The Measurement of Highlights in Color Images. International Journal of Computer Vision 2 (1988), 7-32.

[34] Koenderink, J. J., And van Doorn, A. J. Surface shape and curvature scales. Image Vision Computing 10, 8 (1992), 557-565.

[35] LAND, E., AND MCCANN, J. Lightness and retinex theory. Journal of Optical Society America 61, 1 (1971), 1-11.

[36] LAND, E. H. Recent advances in retinex theory. Vision Research 26, 1 (1986).

[37] LEE, H.-C. Method for computing the scene-illuminant chromaticity from specular highlights. Journal of the Optical Society of America A 3 (1986), 1694-1699.

[38] LI, S. Z. Discontinuous mrf prior and robust statistics: a comparative study. Image Vision Computing 13, 3 (1995), 227-233.

[39] Lin, S., AND Shum, H.-Y. Separation of Diffuse and Specular Reflection in Color Images. Computer Vision and Pattern Recognition, IEEE Computer Society Conference on 1 (2001), 341.

[40] Mallick, S. P., Zickler, T., Belhumeur, P. N., And Kriegman, D. J. Specularity Removal in Images and Videos: A PDE Approach. In ECCV (1) (2006), pp. 550-563.

[41] Narasimhan, S. G., Ramesh, V., And Nayar, S. K. A Class of Photometric Invariants: Sepa- 
rating Material from Shape and Illumination. In International Conference on Computer Vision (Washington, DC, USA, 2003), IEEE Computer Society, pp. 1387-1394.

[42] NAYAR, S., AND BOLLE, R. Reflectance based object recognition. International Journal of Computer Vision 17, 3 (1996), 219-240.

[43] Nayar, S. K., AND Bolle, R. M. Computing reflectance ratios from an image. Pattern Recognition 26 (1993), 1529-1542.

[44] Phung, S. L., Bouzerdoum, A., And Chai, D. Skin segmentation using color pixel classification: Analysis and comparison. IEEE Trans. Pattern Anal. Mach. Intell. 27, 1 (2005), 148-154.

[45] Poggio, T., And Torre, V. Ill-posed problems and regularization analysis in early vision. Tech. rep., Cambridge, MA, USA, 1984.

[46] Ragheb, H., And Hancock, E. R. Highlight removal using shape-from-shading. In European Conf. on Comp. Vision (2002), no. 2351 in LNCS, pp. 626-641.

[47] Raskar, R., Tumblin, J., Mohan, A., Agrawal, A., And Li, Y. Computational Photography. In Proceeding of Eurographics: State of the Art Report (STAR).

[48] Schaefer, G., Hordley, S., And Finlayson, G. A Combined Physical and Statistical Approach to Colour Constancy. Computer Vision and Pattern Recognition, IEEE Computer Society Conference on 1 (2005), 148-153.

[49] ShAFER, S. A. Using color to separate reflection components. Color Research and Applications 10, 4 (1985), 210-218.

[50] Slater, D., And Healey, G. Object recognition using invariant profiles. In Computer Vision and Pattern Recognition (1997), pp. 827-832.

[51] Stockman, H., And Gevers, T. Detection and classification of hyperspectral edges. In British Machine Vision Conference (1999), pp. 643-651.

[52] Suen, P. H., And Healey, G. Invariant mixture recognition in hyperspectral images. In Int. Conference on Computer Vision (2001), pp. 262-267.

[53] Sunshine, J. M., Pieters, C. M., And Pratt, S. F. Deconvolution of Mineral Absorption Bands: An Improved Approach. Journal of Geophysical Research 95, B5 (1990), 6955-6966.

[54] TAN, R. T., Nishino, K., AND IKEUChI, K. Separating reflection components based on chromaticity and noise analysis. IEEE Trans. Pattern Anal. Mach. Intell. 26, 10 (2004), 1373-1379.

[55] Tominaga, S., And Wandell, B. A. Standard surface-reflectance model and illuminant estimation. Journal of the Optical Society of America A 6 (April 1989), 576-584.

[56] Tominaga, S., And WAndell, B. A. Component estimation of surface spectral reflectance. Journal of the Optical Society of America A 7, 2 (February 1990), 312-317.

[57] VAN DE WEIJER, J., AND GEVERS, T. Color constancy based on the grey-edge hypothesis. In IEEE 
International Conference on Image Processing (2005), vol. 2, pp. 722-725.

[58] Wang, J., AND DANA, K. J. Relief texture from specularities. IEEE Transactions on Pattern Analysis and Machine Intelligence 28, 3 (2006), 446-457.

[59] Worthington, P. L., And Hancock, E. R. New constraints on data-closeness and needle map consistency for shape-from-shading. IEEE Transactions on Pattern Analysis and Machine Intelligence 21, 12 (1999), 1250-1267.

[60] Zheng, Q., AND Chellapa, R. Estimation of illuminant direction, albedo, and shape from shading. IEEE Transactions on Pattern Analysis and Machine Intelligence 13, 7 (1991), 680-702.

[61] Zickler, T., Mallick, S. P., Kriegman, D. J., And Belhumeur, P. N. Color subspaces as photometric invariants. International Journal of Computer Vision 79, 1 (2008), 13-30. 